\title{
The stress-sensing domain of activated IRE1 $\alpha$ forms helical filaments in narrow ER membrane tubes
}

\author{
Stephen D. Carter ${ }^{1 \dagger \#}$, Ngoc-Han Tran ${ }^{2 \dagger \#}$, Ann De Mazière ${ }^{3}$, Avi Ashkenazi ${ }^{4}$, Judith \\ Klumperman $^{3}$, Grant J. Jensen ${ }^{1,5^{*}}$, Peter Walter ${ }^{2,6^{* \#}}$
}

\begin{abstract}
${ }^{1}$ Division of Biology and Biological Engineering, California Institute of Technology, Pasadena, CA, USA; ${ }^{2}$ Department of Biochemistry and Biophysics, University of California, San Francisco, CA, USA; ${ }^{3}$ Section Cell Biology, Center for Molecular Medicine, University Medical Center Utrecht, Utrecht, Netherlands; ${ }^{4}$ Cancer Immunology, Genentech, Inc., South San Francisco, CA, USA, ${ }^{5}$ Department of Chemistry \& Biochemistry, Brigham Young University, Provo, UT, USA; ${ }^{6}$ Howard Hughes Medical Institute, University of California at San Francisco, San Francisco, CA, USA
\end{abstract}

*Correspondence to: G.J.J.: jensen@caltech.edu; P.W.: peter@walterlab.ucsf.edu

${ }^{\dagger}$ Authors contributed equally

\# In the spirit of equal contribution, the order of co-first and co-corresponding authors will be switched for publication. Author order was determined by coin flip.

The signaling network of the unfolded protein response (UPR) adjusts the protein folding capacity of the endoplasmic reticulum (ER) according to need. The most conserved UPR sensor, IRE1 $\alpha$, spans the ER membrane and activates through oligomerization. IRE1 $\alpha$ oligomers accumulate in dynamic foci. We determined the in-situ structure of IRE1 $\alpha$ foci by cryogenic correlated light and electron microscopy (cryo-CLEM), combined with electron cryo-tomography (cryo-ET) and complementary immunoelectron microscopy. IRE1 $\alpha$ oligomers localize to a network of narrow anastomosing ER tubes (diameter $\sim 28 \mathrm{~nm}$ ) with complex branching. The lumen of the tubes contains protein filaments, likely composed of linear arrays of IRE1 $\alpha$ lumenal domain dimers, arranged in two intertwined, left-handed helices. Our findings define a previously unrecognized ER subdomain and suggest positive feedback in IRE1 signaling.

\section{Introduction}

The vast majority of secreted and transmembrane proteins mature in the endoplasmic reticulum (ER), which provides a specialized folding compartment with unique biochemical and proteomic characteristics (1). To ensure proper folding, a network of quality control pathways, collectively termed the unfolded protein response (UPR), continuously monitors ER folding status and adjusts its capacity according to cellular demand (2). The three branches of the metazoan UPR, named after their pivotal ER-resident sensors (IRE1 (3, 4), PERK, and ATF6), activate in response to disruptions in protein folding homeostasis, characterized by an accumulation of unfolded proteins within the ER lumen. This condition, known as ER stress, triggers corrective cellular measures or, if the defect cannot be corrected, apoptotic cell death (5).

The life/death decision made after UPR activation involves a molecular timer in which IRE1 activation initially provides cytoprotective outputs but then attenuates even under conditions where ER stress remains unmitigated (6). IRE1 attenuation thus predisposes the cell to apoptosis as a consequence of persistent, unopposed PERK signaling (7, $8)$. Therefore, IRE1 signaling in 
particular and UPR signaling in general sit at a junction of cellular homeostasis and cell death. Maladaptive UPR signaling is a hallmark of many diseases, including cancer, diabetes, and neurodegeneration (9).

Mammalian IRE1 has two paralogs (10), IRE1 $\alpha$ and IRE1 $\beta$. IRE1 $\alpha$ is the major isoform expressed in most cell types. It is an ERtransmembrane protein bearing an ER-stress sensing domain on the ER lumenal side and kinase/ribonuclease (RNase) effector domains on the cytosolic side $(3,4,11)$. IRE1 $\alpha$ 's lumenal domain (LD) is bound by the ERlumenal HSP70-like chaperone BiP, which dissociates upon ER stress (12). IRE1 $\alpha$-LD then binds directly to accumulated unfolded proteins, which triggers its oligomerization (13, 14). Oligomerization of the LD in turn drives the juxtaposition of IRE1 $\alpha$ 's cytosolic kinase/RNase domains, which activate after trans-autophosphorylation (15). Lipid stress can likewise induce IRE1 activation through oligomerization, bypassing a need for the LD $(16,17)$. IRE1 $\alpha$ 's activated RNase domain initiates the non-conventional splicing of its substrate XBP1 mRNA (18). Spliced XBPI mRNA is translated to produce the potent transcription factor XBP1s that upregulates hundreds of genes to restore ER homeostasis. A second consequence of IRE1 $\alpha$ RNase activation, termed RIDD (regulated IRE1dependent mRNA decay), is the selective degradation of a spectrum of ER-bound mRNAs (19). RIDD reduces the protein folding burden in the ER and initiates other protective effects $(7,20)$, thus synergizing with $X B P 1$ mRNA splicing to alleviate ER stress and preserve cell viability.

Upon UPR induction, a fraction of IRE1 molecules cluster into large oligomers that are visible as discrete foci by fluorescence microscopy $(21,22)$. This extensive oligomerization is consistent with the observation that IRE1's lumenal and cytosolic domains form dimers and higher-order oligomers in vitro, also observed in various crystal structures (23-25). Both oligomerization of the kinase/RNase domains in vitro and formation of foci in cells correlate with high enzymatic activity (25).

We and others have shown that IRE1 $\alpha$ foci are entities with complex morphology and dynamic behaviors $(26,27)$. Foci comprise two distinct populations of IRE1 $\alpha$ (27). A small fraction of clustered molecules rapidly exchanges with the pool of dispersed IRE1 $\alpha$ in the ER membrane, while the majority are diffusionally constrained to a particular cluster and remain there until that cluster's eventual dissolution. When foci disappear at late timepoints of stress, their constituent IRE1 $\alpha$ molecules are efficiently recycled back into the ER network rather than degraded. The foci are therefore not phaseseparated liquid condensates but resemble higher-order arrangements whose assembly and disassembly are likely regulated by distinct mechanisms. However, the molecular principles that explain IRE1 $\alpha$ 's complex dynamics during ER stress and the functional consequences of its different assembly states remain a mystery.

\section{Results}

\section{IRE1 $\alpha$ oligomers localize to specialized ER regions with complex topology}

Leveraging recent advances in in situ structural determination of protein complexes in their native cellular environment $(28,29)$, we applied cryoCLEM to determine the ultrastructure of IRE1 $\alpha$ foci in mammalian cells. To this end, we constructed stable cell lines that express fluorescently tagged human IRE1 $\alpha$ under control of an inducible promoter $(14,27)$. We grew cells directly on fibronectin- or collagen-coated gold EM grids to $15 \%$ confluency and induced IRE1 $\alpha$ expression. We next promoted ER stress with tunicamycin, which blocks N-linked protein glycosylation in the ER lumen, thereby activating IRE1 $\alpha$. We added blue fluorescent nanospheres to the grids as positional markers and plunge-froze 


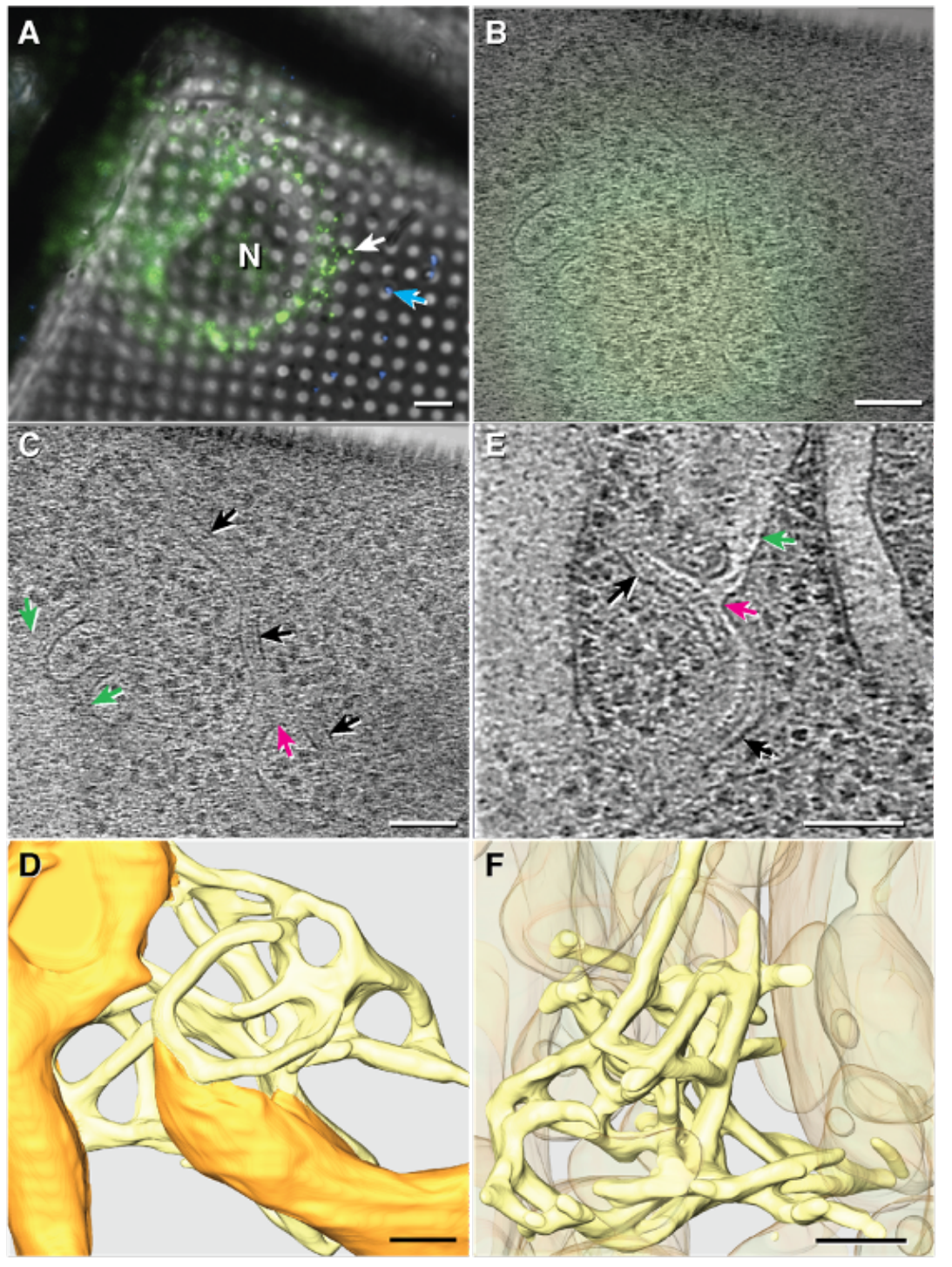

Figure $1 \mid$ IRE1 $\alpha$ oligomers localize to specialized ER regions with complex topology. (A) Fluorescent profiles imaged at liquid nitrogen temperature for stressed cells grown on EM grids and expressing IRE1 $1 \alpha-m N G$. White arrow denotes the fluorescent spot corresponding to the tomogram depicted in $(B-D)$. Blue arrow: $500 \mathrm{~nm}$ fluorescent nanosphere. " $N$ ": Nucleus. Scale bar $=6 \mu \mathrm{m}$. (B) Correlation of fluorescence image with a representative $z$ slice $(C)$ of the tomogram showing examples of narrow membrane tubes (black arrows) connected to general ER network at junctions (green arrows) and to each other at three-way junctions (magenta arrows). The tomogram was manually segmented for $3 D$ visualization in $(D)$, where normal ER membranes are depicted in orange and constricted membranes colocalizing with IRE1 $\alpha-m N G$ signal are yellow. (E) A representative $z$ slice from a higher resolution tomogram obtained in stressed U2OS-IRE1 $\alpha$-mNG cells. (arrows color code same as (C)). (F) manual segmentation of region shown in $(E)$ with constricted membranes shown in yellow and other membranes shown in orange at $50 \%$ transparency. Scale bars for $B-F=100 \mathrm{~nm}$. Densities corresponding to ribosomes and cytoplasmic densities in $(D)$ and $(F)$ are omitted for clarity. See Fig. S4.

the samples in a mixture of liquid ethane/propane at $77 \mathrm{~K}(30)$.

To localize IRE1 $\alpha$ foci, we first imaged the frozen grids on a fluorescent light microscope fitted with a liquid nitrogen sample chamber (31). In our initial studies with an IRE1 $\alpha$-GFP fusion protein, the stressed cells exhibited strong autofluorescence at liquid nitrogen temperature $(77 \mathrm{~K})$ as previously observed (32), which hindered identification of IRE $1 \alpha$ foci (Fig. S1). To overcome this hurdle, we fused IRE1 $\alpha$ to the exceptionally bright fluorescent protein mNeonGreen ( $\mathrm{mNG}$ ). This experimental refinement revealed spots emitting high fluorescence in the green but much lower fluorescence in the red and blue channels (Fig.1A). Spots meeting these criteria were absent in control cells expressing IRE1 $\alpha$ not fused to $\mathrm{mNG}$ (Fig. S1), while auto-fluorescent spots with high green, blue and red signals were abundant in both samples. Thus, plotting the ratio of green/red and green/blue fluorescence intensity allowed us to exclude non-specific signals (Fig. S1) in order to identify IRE $1 \alpha-\mathrm{mNG}$ foci reliably.

We next imaged IRE1 $\alpha$ foci by cryo-CLEM combined with cryo-ET. To this end, using nanospheres, grid features, cell boundaries and other landmarks (such as ice), we located the same IRE1 $\alpha$ foci with the electron microscope that we had previously identified with the light microscope and then recorded tilt-series. Across 9 tomograms obtained from mouse embryonic fibroblasts (MEFs), fluorescent IRE1 $\alpha$ foci consistently localized to specialized regions of the ER that display a network of remarkably narrow, 
anastomosing tubes (Fig. 1B$\mathrm{D}$ and Fig. S2-3) with an average diameter of $28 \pm 3$ $\mathrm{nm}$ ( \pm standard deviation). As visualized in segmented three-dimensional reconstructions, the tubes frequently connect with each other by three-way junctions and to surrounding ER structures, forming a topologically complex yet continuous membrane surface (Fig. 1D, Supp Fig. S2-3). Unlike the surrounding ER, the narrow anastomosing tubes colocalized with IRE $1 \alpha$ foci are devoid of bound ribosomes (Fig. S4).

To confirm these results and obtain higher resolution images, we used human osteosarcoma U-2 OS cells that likewise express inducible IRE1 $\alpha-\mathrm{mNG}$ (27). Compared to MEFs, U-2 OS cells spread more and therefore contain expansive thin regions that enhance the resolution of cryo-ET imaging. We imaged tilt series from 8 IRE $1 \alpha-m N G$ foci at slightly higher magnification $(33,000 \mathrm{x}$ for U-2 OS cells vs. 22,000x for MEFs). We again observed thin anastomosing tubes with similar characteristics as those in MEFs, including the absence of bound ribosomes, three-way junctions and connections to adjacent ER (Fig. 1E-F and Fig. S4-5). Using neural networkenhanced machine learning to segment the tomograms (33), we confirmed the basic features of the manual 3D reconstructions without subjective bias (Fig. S6). Taken together, IRE1 $\alpha$ foci localize to a highly specialized ER region, henceforth termed the "IRE1 $\alpha$ subdomain".

\section{Orthogonal methods reveal IRE1 $\alpha$ subdomains}

To validate our discovery of IRE1 $\alpha$ subdomain tubes with alternative approaches, we performed conventional and immunoelectron microscopy on HEK293 cells expressing IRE1 $\alpha$-GFP fusion protein (22). In ER-stressed HEK293 cells, electron micrographs of chemically fixed, stained and Epon-embedded thin sections exhibit thin membrane tubes and networks of comparable topology to those seen in the cryo-tomograms (Fig. S7) and unlike anything seen in un-stressed, control cells. These structures are infrequently observed and stained more strongly in their lumenal space than the surrounding ER, suggesting the presence of a high protein density inside and likely represent IRE1 $\alpha$ subdomains.

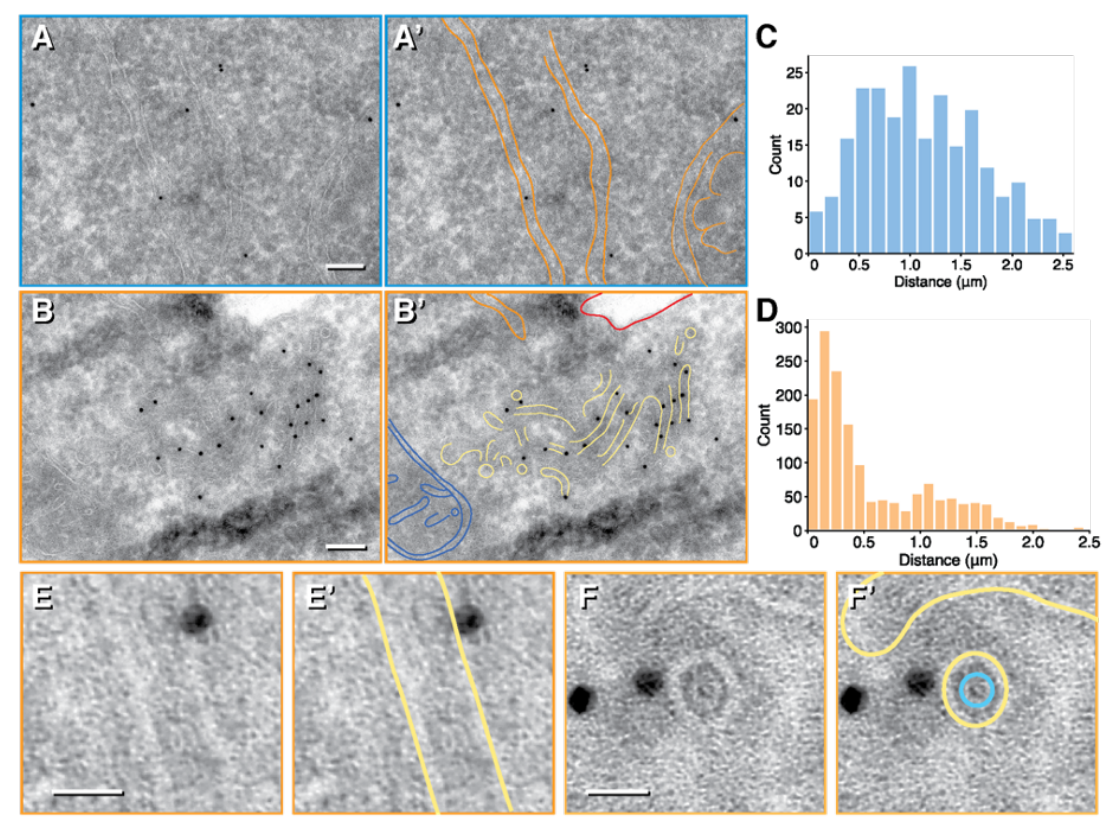

Figure 2| Orthogonal immuno-electron microscopy reveals IRE1 $\alpha$ subdomains. (A, $A$ ') Representative micrograph of cells expressing IRE1 $\alpha-G F P$ but not subjected to ER stress induction by Tunicamycin (Tm). Gold particles recognizing IRE1 $\alpha$-GFP epitope (via binding to anti-GFP primary antibody) sparsely label general ER structures. Scale bar $=100 \mathrm{~nm}$. In A', orange: ER sheet/tubule membranes (B, B') Representative micrograph of cells treated with Tm where gold particles recognizing IRE1 $\alpha-G F P$ epitope densely localizes to a region enriched in narrow membranes of $26 \pm 2 \mathrm{~nm}$ diameter. Scale bar $=100 \mathrm{~nm}$. In B', blue: mitochondrion, orange: ER sheet/tubule membranes, red: plasma membrane, yellow: narrow IRE1 a subdomain membranes. Histograms of inter-gold particle distances measured in micrographs from non-stressed samples $(C)$ and stressed samples (D) reveal a population of densely clustered gold particles enriched with ER stress induction. Zoomed in view showing longitudinal (E, $\left.E^{\prime}\right)$ and end-on $\left(F, F^{\prime}\right)$ tube cross-sections with $\sim 28 \mathrm{~nm}$ diameter close to gold particles. Scale bar $=20 \mathrm{~nm}$. A ring-like density within the lumenal space is clearly visible in $F$ (segmented in teal) 
To directly identify IRE1 $\alpha$ foci in electron micrographs, we next performed immunogold labeling of ultrathin cryosections of HEK293 cells expressing IRE1 $\alpha$-GFP. As above, we induced ER stress with tunicamycin to drive activated IRE1 $\alpha$ into foci. In non-stressed cells, gold particles specific to IRE1 $\alpha$-GFP sparsely label large regions of the cell with visible ER (Fig. 2A-A' and Fig. S8). By contrast, in stressed cells we observed clusters of gold particles in regions of much higher membrane complexity (Fig. 2B-B', 2E-F'and Fig. S8). In these regions, we observed narrow membrane tubes of similar diameter $(\sim 28 \mathrm{~nm})$ as both longitudinal and transverse cross sections. Quantification of the inter-particle distances between samples revealed a clear difference in gold particle density (Fig. 2C-D), reflecting a population of clustered IRE1 $\alpha$ GFP molecules in stressed cells that is absent in non-stressed control cells. This observation is consistent with previous data that show that IRE1 $\alpha$ molecules, which uniformly distributed in the ER during homeostasis, aggregate in foci of dozens of IRE $1 \alpha$ molecules during ER stress induction $(22,26,27)$. Notably, we observed a distinct lumenal density inside the membrane tubes, which is circular in transverse cross sections (Fig. 2E-F').

\section{IRE1 $\alpha$ subdomain membrane tubes contain lumenal helical filaments.}

Consistent with the density observed in immunogold labeling experiments, we observed regular densities in the lumen of the IRE1 $\alpha$ subdomain tubes in cryogenic tomographic reconstructions, which we interpret as oligomers of IRE $1 \alpha-L D$. In our MEF-derived tomograms, the lumenal densities resemble train tracks that in longitudinal sections run parallel to the membranes (Fig. 3A-A'). Closed rings roughly concentric with the enclosing tube membrane are clearly visible in instances where IRE1 $\alpha$ subdomain tubes are imaged parallel to the beam direction (Fig. 3B-B' and Fig. S9). The inner rings measure $9 \pm 0.5 \mathrm{~nm}$ in diameter and are enclosed by membrane tubes that are approximately $28 \pm 1 \mathrm{~nm}$ diameter (Fig. 3C).

Strikingly, in the tomograms of U-2 OS cells, the lumenal densities show sufficient substructure to reveal two intertwined helices (Fig. 3D-F'). This helical feature is most clearly seen in IRE1 $\alpha$ subdomain tubes in which the top and bottom cross sections show equidistant parallel angled lines of opposite directionalities, whereas the middle cross section shows helical features (Fig. 3D-G).

\section{Sub-tomogram averaging resolves flexible IRE1 $\alpha$-LD double helices}

To determine the 3D structure of the lumenal double-helical density, we extracted subvolumes along the membrane tubes for

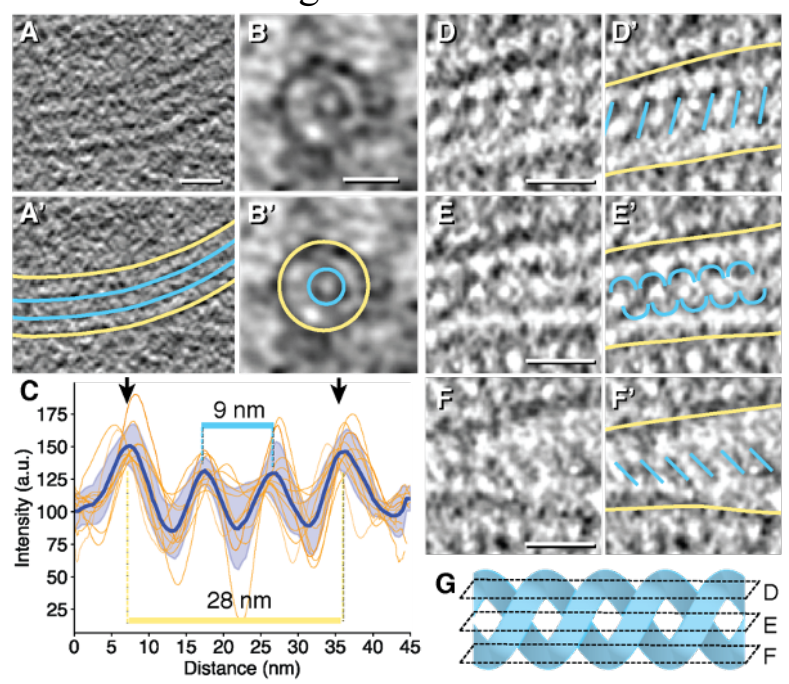

Figure 3 IRE1a subdomain membrane tubes contain lumenal protein densities. Representative examples of a longitudinal cross-section ( $\left.A, A^{\prime}\right)$ and an end-on cross-section $\left(B, B^{\prime}\right)$ obtained in MEFs-IRE1-mNG cells revealing membrane density (yellow) surrounding lumenal protein density (teal). Scale bar $=20 \mathrm{~nm}$. Intensity line plots across subdomain tubes are aligned, plotted and averaged across 9 cross-sections and plotted as a function of distance in $(C)$. Blue line with shaded error of the mean is the averaged trace for all plots. Distance separation of peak maxima are indicated for peaks representing membrane densities (yellow) and those representing protein densities (teal). ( $D-F$ ') show an example of lumenal protein densities with helical features obtained in U2OS-IRE1-mNG cells viewed as top (D, D'), middle (E, $\left.E^{\prime}\right)$ and bottom $\left(F, F^{\prime}\right)$ sections. Scale bars $=20 \mathrm{~nm}$. G) Schematic of an idealized double helix to illustrate how the cryotomogram slices in $D-F$ would intercept as planes and give rise to the corresponding densities segmented in $D^{\prime}-F$ '. 
subtomogram averaging, using the EMAN2 tomogram processing workflow (Fig. S10). The resulting electron density map obtained by averaging of 653 subvolumes portrayed a double helix composed of two equidistant intertwined strands with a pitch of $17 \mathrm{~nm}$ in each individual strand (Fig. 4A, B and Fig. S10). Analysis of sequential cross sections revealed that the double helices are left-handed (Fig. 4A). The handedness was confirmed by subtomogram averaging of ribosomes in the same tomograms (data not shown). This double helical structure is reminiscent of the unit cell of the $S$. cerevisiae IRE1-LD crystal structure ((23) PDB ID: 2BE1), in which twofold symmetrical head-to-head IRE1-LD dimers arrange into helical filaments by forming tail-to-tail contacts. Two such yeast IRE1-LD filaments intertwine in left-handed double helices.

With fluorescence, immunogold labeling, and structural (lefthanded intertwined helices) evidence that the regular density in the tubes is IRE1 $\alpha-\mathrm{LD}$, we endeavored to interpret its structure in light of existing atomic models. The two most relevant structures are (i) the crystal structure of the active, polymeric yeast IRE1-LD, which forms intertwined left-handed helices like those in the cryo-tomograms but with different pitch (38 $\mathrm{nm}$ ); and (ii) the crystal structure of the human IRE1 $\alpha$-LD in

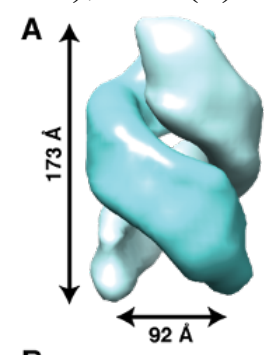

B

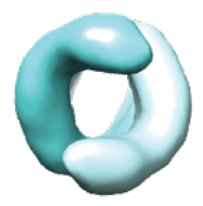

C

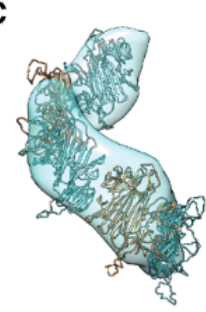

D

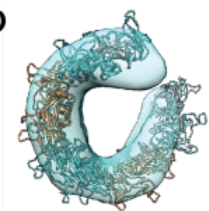

E
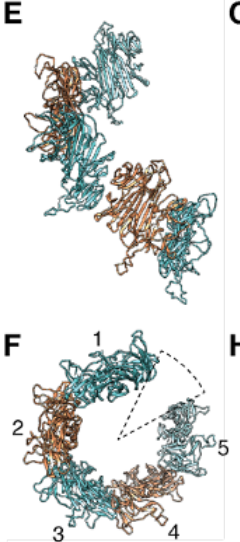
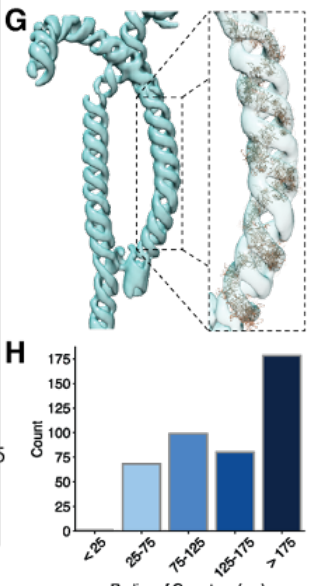

Figure 4 |Sub-tomogram averaging resolves flexible IRE1a-LD double helices. (A-B) Electron density map obtained by sub-tomogram averaging of 653 subvolumes in U2OS-IRE1-mNG tomograms with indicated distances for helical pitch and width. (C, D) Semi-transparent masked average of one strand of the double helix fitted with modeled IRE1 $\alpha$ lumenal oligomer $(E, F)$ using Chimera's "fit-in-map" function. 77\% of the structure fit within this density. (B, D and F) are rotation of $(A, C, E)$ by 90 degree along $X$ axis. $(F) 5$ dimers of IRE $1 \alpha$-cLD are fitted into masked single-strand map, but approximately 6 dimers are required to complete one full turn. (G) An isosurface of the averaged density mapped back on to the cryotomogram at a highly curved region and fitted with a helix of the modeled IRE1a lumenal structure using a range of interface angles to accommodate curvature. (H) A histogram of measured radii of curvature observed for $25 \mathrm{~nm}$ segments along IRE1 $\alpha$ subdomain network. its inactive dimeric form. We chose to begin with the former and used SWISS-MODEL homology modeling to predict a homologous human IRE1 $\alpha$ LD structure based on the yeast structure (Fig. S11) (34). The resulting model retains similarity in secondary and tertiary structure compared to the (inactive) human IRE1 $\alpha$ LD but contains rearranged elements at the tail-ends of the homodimer (Fig. S11-12). We next fit the modeled head-tohead IRE1 $\alpha$-LD dimer into our double-helical map as a rigid body and modified the tail-totail dimer interface so that the polymer would exhibit the same helical pitch observed in the tomograms (Fig. 4C-D and Figs. S13). We maintained both head-to-head and modified tail-to-tail interfaces in propagating the array of dimers to occupy the helical map. The resulting model contains approximately six dimers per turn in each helical filament (Fig. 4E-F), consistent with the $S$. cerevisiae LD crystal structure (Fig. S14) but with a compressed pitch (17 nm vs. $38 \mathrm{~nm}$ in the yeast crystal structure). We conclude that an IRE1 $\alpha$-LD polymer accounts well for the regular density, including in the details of its thin ribbon-like shape. By analogy to the yeast LD structure, we fit the human LD into the density with its putative unfolded protein binding groove facing towards 

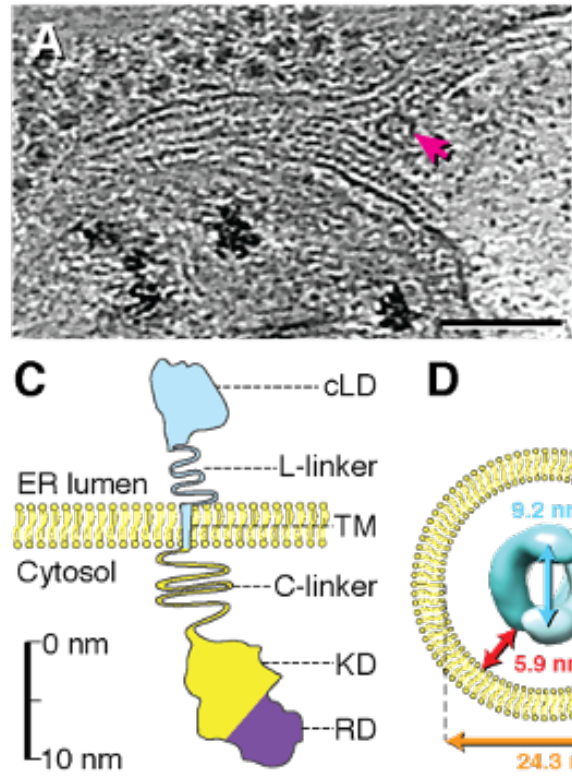
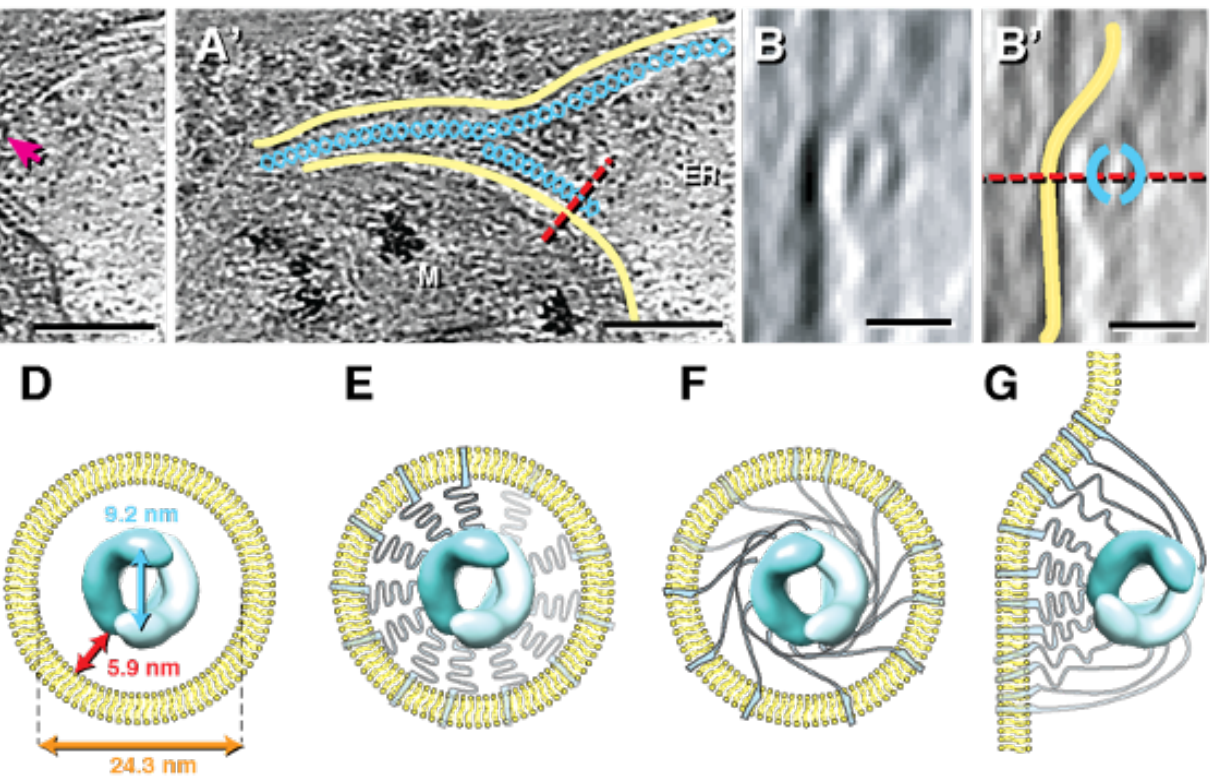

G

Figure 5 | IRE1 $\alpha-L D$ helices can accommodate a range of distance from membrane. $\left(A, A^{\prime}\right)$ An instance of ordered IRE $\alpha$ - $L D$ helices not completely enveloped by narrow membrane tubes. Arrow in A indicates a membrane fenestration. ER: lumenal space; M: mitochondrion. Scale bar $=100 \mathrm{~nm}$. (B, B') Side view along indicated plane obtained by 90-degree rotation along $X$ axis. Scale bar $=20 \mathrm{~nm} .(C)$ Diagram of IRE1 $\alpha$ domain architecture drawn to approximate scale. cLD: core lumenal domain (a.a. 19390), L-linker: lumenal linker (a.a. 391-443), TM: transmembrane helix (a.a. 444-464), C-linker: cytoplasmic linker (a.a. 465570), KD: kinase domain (a.a. 571-832), RD: ribonuclease domain (a.a. 835-964). Scale bar =10 nm. (D) Dimensions of IRE1 $\alpha$ $L D$ helices within IRE1 subdomain lumenal space. (E, F) Schematics for alternative TM domain and L-linker arrangements within the narrow subdomain tubes. There are 24 monomers per turn for the double helices, but only 12 TM and L-linker domains are shown for clarity. (G) Model for TM and L-linker arrangement for helices not completely surrounded by membrane as seen in $B$ the membrane; however, at the current resolution this assignment must be considered tentative because no secondary structural features are resolved that would support this orientation. Likewise, the details of the subunit contacts remain uncertain.

Mapping the averaged subvolumes back onto the cryo-tomograms (Fig. 4G) allowed us to generate a volumetric map for IRE1 $\alpha$-LD within an IRE1 $\alpha$ subdomain. The resulting distribution of double-helical filaments showed that the helices are not stiff but curve to varying degree (Fig. 4H and Fig. S15), ranging from straight segments (radius of curvature $>175 \mathrm{~nm}$ ) to segments bent with radii approaching $25 \mathrm{~nm}$. This bending indicates that IRE1 $\alpha$-LDs, their oligomerization interfaces, and/or inter-strand connections must undergo conformational rearrangements that can accommodate the observed range of curvatures.

\section{IRE1 $\alpha$-LD helices and IRE1 $\alpha$ subdomains are flexible}

Further examination of the tomograms revealed infrequent instances in which lumenal double helices are irregularly spaced from the tubular membranes (Fig. S16). In one case, we observed helices that are not completely enclosed by membrane tubes but instead are positioned on the lumenal face of a flat ER membrane (Fig. 5A-B').

The helically arranged IRE1 $\alpha$ core-LDs (cLD) are each attached via a 52-amino acid linker to IRE1 $\alpha$ 's transmembrane domains, which in turn are connected to the cytosolic kinase/RNase domains (Fig. 5C). In IRE1 $\alpha$ subdomain tubes, the linker domain could either be in a compacted or an extended conformation to bridge the $5.9 \mathrm{~nm}$ distance between the helix and the membrane (Fig. 5D; and Fig. 5E-F, respectively). By contrast, when IRE $1 \alpha$-cLD helices are attached to a flat ER 
membrane surface on one side only as shown in Fig. 5A-B, their linker domains must accommodate the different distance requirements imposed by the positioning of individual IRE1 $\alpha$-cLD monomers to reach the nearest membrane surface, likely conforming to a range of compaction (Fig. 5G). One consequence of this arrangement is that IRE1 $\alpha$ kinase/RNase domains on the cytosolic side of the membrane are brought into even closer proximity and hence experience a higher local concentration (we estimate it likely exceeding $1 \mathrm{mM}$, see Methods) than in the tubes, in which the membrane surface surrounding the helices is $\sim 4$-fold larger than the contact surface of the helices attached to a flat membrane sheet.

\section{Discussion}

The ER is formed from a single continuous membrane that is dynamically differentiated into a plethora of pleomorphic subdomains (35), including the nuclear envelope, smooth tubules, tubular matrices (36), ribosome studded flat sheets, ER exit domains, interorganellar contact sites (37), and ER-phagic whorls (38). Using cryo-CLEM to inspect IRE1 $\alpha$ fluorescent puncta at macromolecular resolution, we found that UPR activation leads to the formation of previously unrecognized "IRE1 $\alpha$ subdomains", comprised of topologically heterogeneous assemblies of anastomosing $\sim 28 \mathrm{~nm}$ membrane tubes. Inside the highly constricted lumenal spaces of this labyrinthine network, IRE1 $\alpha$-LDs assemble into ordered double-helical filaments. Our use of cryo-CLEM and cryo-ET to visualize IRE1 $\alpha$ oligomers directly in intact cells demonstrates the power of in situ structural biology, providing insight into the supramolecular arrangements of molecules in their native environment.

Four independent lines of evidence support our conclusion that the observed helical densities indeed correspond to oligomerized IRE1 $\alpha$ LDs: (i) In 20 out of 20 fluorescent foci analyzed by EM, we observed narrow membrane tubes, which in 18 out of 20 have a consistent diameter of $\sim 28 \mathrm{~nm}$ and contain lumenal protein density consistent with the helical reconstruction. By contrast, no such structures were observed in adjacent and random regions of the cell, including those emitting high autofluorescence; (ii) orthogonal analysis by immunogold-staining of thin sections revealed IRE1 $\alpha$ localization to similarly-narrow tubes; (iii) the double-helical architecture closely resembles that observed in the crystal structure of yeast IRE1-cLD (23); and (iv) the reconstructed helical volume has the same flat ribbon-like shape and dimensions as IRE1 $\alpha$-cLD oligomers.

The accumulation of IRE1 $\alpha$ within these specialized structures explains recent observations that IRE $1 \alpha$ foci contain a readily exchanging periphery and a diffusionally constrained core (27). We surmise that the two distinct populations represent (i) IRE1 $\alpha$ molecules located at the subdomain junctions where the $28 \mathrm{~nm}$ tubes merge with the main ER and (ii) those located deeper in the interior of the narrow tubes. IRE1 $\alpha$ molecules at helix ends located near the tube mouths can readily dissociate, and new IRE1 $\alpha$ molecules can associate; they thus represent a freely exchanging pool at the foci's periphery. By contrast, IRE1 $\alpha$ molecules at the foci's core are physically trapped in regularly arrayed helices; they thus represent a non-exchangeable pool.

The confinement of IRE1 $\alpha$ in the anastomosing IRE1 $\alpha$ subdomains suggests functional consequences for the regulation of IRE1 signaling. The presence of just a single unfolded protein molecule in each $100 \mathrm{~nm}$ long cylinder segment amounts to an effective concentration of $\sim 40 \mu \mathrm{M}$, which is in the same order of magnitude as the affinity measured for IRE1-unfolded protein binding in vitro (13, 14). Thus a few unfolded protein molecules trapped inside the tubes would saturate IRE1 $\alpha$ LD with activating ligand, triggering a positive feedback loop that effectively locks IRE1 $\alpha$ into its activated state. This effect is due to the 
enormous concentration IRE1 $\alpha$ experiences upon foci formation. Without UPR activation, IRE $1 \alpha-\mathrm{mNG}$ is distributed over the ER surface at about 10 molecules per $\mu \mathrm{m}^{2}$ (27). By contrast, inside IRE1 $\alpha$ subdomains, it is enriched 1500 -fold to 15,000 molecules per $\mu \mathrm{m}^{2}$ of ER membrane (Methods). Moreover, based on decreased diffusional freedom due to the complex membrane architecture (39) and IRE1 $\alpha$ LD's helical assembly, IRE1 $\alpha$ subdomains stabilize the oligomeric state. In an IRE1 $\alpha$ subdomain, we estimate that the local concentration of IRE $1 \alpha$ 's LD inside the tubes and IRE1 $\alpha$ cytosolic domains on the tubes' cytosolic surface approaches $5 \mathrm{mM}$ and $200 \mu \mathrm{M}$, respectively. These concentrations well-exceed the range of affinities measured in vitro for purified IRE1 domain oligomerization $(25,40)$.

The resemblance to the crystal structure of the yeast IRE1 cLD is remarkable: this structure likewise reveals two intertwined, equidistant left-handed helices with 12 LDs per turn and strand, albeit 2.2-fold more stretched out along the central axis. It will certainly be interesting to repeat this work with yeast cells to see if the differences are species-specific or due to artefacts of crystallization conditions. Given that two helices of the IRE1 $\alpha$-LDs are equidistantly intertwined, there may be bridges between them, perhaps formed from unresolved regions of the cLD or linker domains, and/or bound unfolded protein chains. We find it equally remarkable that IRE $1 \alpha$ helices are observed both in the narrow membrane tubes of the IRE $1 \alpha$ subdomain as well as, albeit more rarely, lying flat on the lumenal side of ER sheets. Thus, the lumenal IRE1 $\alpha$ linker domains must accommodate a range of distances separating IRE1 $\alpha$-cLD from the membrane surface. These two topologically distinct arrangements of IRE1 $\alpha$ cLD helices may co-exist or could be temporal precursors of one another.

IRE1 $\alpha$ subdomains could form by IRE1 $\alpha$-LD filaments pushing finger-like ER membrane protrusions, which then fuse with adjacent ER, or from IRE1 $\alpha$-LD filaments pushing ridgelike deformations into flat ER membranes, which then separate from the flat ER following membrane fission/fenestration (41). Alternatively, IRE1 $\alpha$ filament polymerization could constrict existing ER tubes to form IRE1 $\alpha$ subdomains or stabilize pre-existing narrow membrane tubes (42) into subdomain tubes with regular $\sim 28 \mathrm{~nm}$ diameter. In support of the latter model, in 2 out of 20 of our tomograms, we observed strong IRE1 $\alpha-\mathrm{mNG}$ signal in regions with thin and irregular membrane tubes, but no ordered lumenal filaments (Fig. S16). Such structures may represent an intermediate state, captured after IRE1 $\alpha$ 's preferential localization but preceding helix formation.

Our discovery of the IRE1 $\alpha$ subdomain raises intriguing possibilities with regard to how recruitment of IRE1 $\alpha$ into these highly specialized ER structures could serve regulatory functions in the UPR. Its high concentration into long-lived topologically distinct structures may scaffold assembly of downstream effectors, as previously proposed (9), and/or affect the selection of IRE1 $\alpha$ mRNA substrates in switching between XBP1 splicing and RIDD activities (19). Such regulation may thus profoundly affect the life/death decision that the UPR makes in response to a breakdown in ER protein folding homeostasis and hence be of crucial importance in designing UPR-centered therapies in protein folding disorders.

\section{Acknowledgements}

We thank Margaret Elvekrog, Ariane Briegel, Diego Acosta-Alvear, Richard Fetter, Suzanne van Dijk, Stefan Niekamp and Vladislav Belyy for their advice and technical assistance. We thank Greg Huber, Robert Ernst, Adam Frost and Jodi Nunnari for insightful discussions.

\section{Funding}


This work was supported in parts by $\mathrm{NIH}$ grants P50 AI150464 and R35 GM122588 to GJJ and NIH R01-GM032384 to PW. NHT is supported by the National Science Foundation Graduate Research Fellowship. JK is supported by the Dutch Research Council NEMI research program project number 184.034.014. ADM received salary from Genentech, Inc. PW is an Investigator of the Howard Hughes Medical Institute.

\section{Author contributions}

AA, JK, PW and GJ, conceived of the study. NHT, SDC, PW and GJ designed cryo-CLEM experiments. NHT and SDC generated and screened grids. SDC performed the cryoCLEM/cryo-ET and subtomogram averaging and generated IRE1 $\alpha$ subdomain models, neural network-derived segmentations and movies. NHT performed manual segmentation, quantification of IRE1 $\alpha$ subdomain dimension and calculations. ADM performed Epon-embedded electron microscopy and immunogold labeling experiments. NHT and SDC prepared figures. NHT, SDC, PW and GJ wrote the manuscript.

\section{Competing interests}

The authors declare no competing interests.

\section{Data and materials availability}

The codes used for data quantification and plots in this paper are freely available at https:/github.com/han-tran/IRE1. The final sub-tomogram averaged maps and representative tomograms can be accessed at EMDB entry ID EMD-23058. All raw and processed data are available upon request. All cell lines and plasmids used in this paper are in the Walter lab depository and are available upon request.

\section{Materials and Methods}

\section{Cell culture and grid preparation}

Previously described MEFs-IRE1-mNG and U2OS-IRE1-mNG cell lines $(14,27)$ were cultured in high glucose DMEM media supplemented with $10 \%$ tetracycline-free fetal bovine serum (FBS; Takara Bio), $6 \mathrm{mM} \mathrm{L-}$ glutamine, and $100 \quad \mathrm{U} / \mathrm{ml}$ penicillin/streptomycin. All cell lines used in the study tested negative for mycoplasma contamination when assayed with the Universal Mycoplasma Detection Kit (ATCC 30-1012K). To minimize autofluorescence, the same culture media without phenol red was used to grow cells for grid preparation (32). Prior to cell plating, gold Quantifoil London finder grids (EMS R2/2 LF-Au-NH2) were UV treated to sterilize and coated with cell adhesion matrix. For MEFs-IRE1-mNG cell line, grids were coated in droplets of 500 $\mu \mathrm{g} / \mathrm{mL}$ Fibronectins (Sigma-Aldrich S5171$.5 \mathrm{MG}$ ) for 5 minutes on each side, washed in PBS, blotted and air-dried. For U2OS-IRE1$\mathrm{mNG}$ cell line, grids were coated with $\sim 4$ $\mathrm{mg} / \mathrm{mL}$ undiluted Collagen type I (Corning 354236) droplets for 20 minutes, washed in PBS, blotted and air-dried. Cells were seeded at $15 \%$ confluence and allowed to adhere for 8 hours and induced with Doxycycline $(500 \mathrm{nM})$ for 6 or 18 hours for MEFs and U-2 OS cell lines, respectively. ER stress was then induced by treatment with $1.5 \mu \mathrm{g} / \mathrm{mL}$ of Tunicamycin for 2 hours.

Immediately prior to being plunge frozen, $3 \mu \mathrm{l}$ of a beads suspension was added to the grids. The bead suspension was made by a 1:1 dilution of $500 \mathrm{~nm}$ blue $(345 / 435 \mathrm{~nm})$ polystyrene fluorospheres (Phosphorex) with a $3: 1$ concentrated solution of $20 \mathrm{~nm}: 5 \mathrm{~nm}$ colloidal gold (Sigma Aldrich) blocked with bovine serum albumin. The gold beads served as fiducial markers for cryo-tomogram reconstruction while the blue fluorospheres served as landmarks for registering cryogenic fluorescence microscopy images collected from different channels as well as with cryoEM projection images for cryo-CLEM (32). Residual media and bead suspension were blotted manually from the back side with Whatmann paper \#40 in 90\% humidity. Grids 
are plunge-frozen in liquid ethane/propane mixture using a Vitrobot Mark IV (FEI, Hillsboro, OR). Plunge-frozen grids were subsequently loaded into Polara EM cartridges (FEI) (31). Cryo-EM cartridges containing frozen grids were stored in liquid nitrogen and maintained at $\leq-150{ }^{\circ} \mathrm{C}$ throughout the experiment including cryogenic fluorescence microscopy imaging, cryo-EM imaging, storage and transfer.

\section{Fluorescence imaging and image processing}

The EM cartridges were transferred into a cryo-FLM stage (FEI Cryostage), modified to hold Polara EM cartridges (31), and mounted on a Nikon Ti inverted microscope. The grids were imaged using a $60 \mathrm{X}$ extra-long-workingdistance air-objective (Nikon CFI S Plan Fluor ELWD 60X NA 0.7 WD $2.62-1.8 \mathrm{~mm}$ ). Images were recorded using a Neo $5.5 \mathrm{sCMOS}$ camera (Andor Technology, South Windsor, CT) using a 2D real-time deblur deconvolution module in the NIS Elements software from AutoQuant (Nikon Instruments Inc., Melville, NY). The 2D real-time deconvolution algorithm estimates a PSF using several factors such as sample thickness, noise levels in the image, background subtraction and contrast enhancement. All fluorescence images (individual channels) were saved in 16-bit grayscale format. IRE $1 \alpha-\mathrm{mNG}$ was visualized with a FITC filter. Blue fluorospheres were visualized with a DAPI filter. Red autofluorescence was imaged using an mCherry filter.

\section{EM imaging}

Cryo-EM grids previously imaged by cryo-LM were subsequently imaged by electron cryotomography using a FEI G2 Polara $300 \mathrm{kV}$ FEG TEM (FEI) equipped with an energy filter (slit width $20 \mathrm{eV}$ for higher magnifications; Gatan, Inc.), and a $4 \mathrm{k} \times 4 \mathrm{k}$ K2 Summit direct detector (Gatan, Inc.) in counting mode.

First, cellular areas containing the fluorescent bodies of interest in suitably-thin areas that were typically 200-500 $\mathrm{nm}$ thick were located in the TEM using methods described previously. Tilt series were then recorded of these areas using UCSF Tomography (43) or SerialEM (44) software at a magnification of $27,500 \times$ (Polara) and 34,000× (Polara). This corresponds to a pixel size of $3.712 \AA$ (MEFs) or $3.260 \AA$ (U2O2), respectively, at the specimen level and was found to be sufficient for this study. Each tilt series was collected from $-60^{\circ}$ to $+60^{\circ}$ with an increment of $1^{\circ}$ in an automated fashion at $4-10 \mu \mathrm{m}$ underfocus. The cumulative dose of one tilt-series was between 80 and $150 \mathrm{e}-/ \AA^{2}$. The tilt series was aligned and binned by 4 into $1 \mathrm{k} \times 1 \mathrm{k}$ using the IMOD software package (45), and 3D reconstructions were calculated using the simultaneous reconstruction technique (SIRT) implemented in the TOMO3D software package (46), or weighted back projection using IMOD. Noise reduction was performed using the non-linear anisotropic diffusion (NAD) method in IMOD (45), typically using a $\mathrm{K}$ value of $0.03-0.04$ with 10 iterations.

\section{Segmentation and isosurface generation}

Segmentation and isosurface rendering were performed in Amira (Thermo Scientific). For lower resolution tomograms from MEFs cells, the segmentation was done all manually using the brush, lasso, and thresholding tools combined with interpolation and surface smoothing. For higher resolution tomograms from U2-OS cells, the ER, IRE1 $\alpha$ subdomains and vesicles were segmented manually. Cytoskeletal components and ribosomes were segmented using the TomoSeg CNN module in EMAN2 (33).

\section{Conventional electron microscopy}

HEK293 stable cells expressing IRE1-GFP, unstressed or treated with tunicamycin, were fixed with $2.5 \%$ glutaraldehyde, 2\% paraformaldehyde in $0.1 \mathrm{M}$ Sorenson's phosphate buffer (PB), $\mathrm{pH} 7.4$, for $2 \mathrm{~h}$ at room temperature. After storage in $1 \%$ PFA, $0.1 \mathrm{M}$ $\mathrm{PB}$ at $4{ }^{\circ} \mathrm{C}$ for about 1 week and rinsing in 0.1 
$\mathrm{M} P B$, the cells were postfixed with $1 \% \mathrm{OsO}_{4}$ and $1.5 \% \mathrm{~K}_{3} \mathrm{Fe}(\mathrm{CN})_{6}$ in $0.07 \mathrm{M} \mathrm{PB}$, stained en bloc in aqueous $0.5 \%$ uranyl acetate, dehydrated in acetone and embedded in Epon. Ultrathin plastic sections were stained with uranyl acetate and lead citrate and examined in a JEOL JEM-1010 electron microscopy. Quantification of the diameters of IRE1a subdomain tubes and ER cisterns was performed using Fiji software (47).

\section{Immuno-electron microscopy}

HEK293 cells stably expressing IRE1-GFP, untreated or treated with tunicamycin, were fixed using $4 \%$ PFA in $0.1 \mathrm{M} \mathrm{PB}$ for $2 \mathrm{~h}$ at room temperature, then overnight at $4{ }^{\circ} \mathrm{C}$. Subsequently, the fixation was continued by replacing the initial fixative with $1 \%$ PFA in $0.1 \mathrm{M}$ PB for several days. The samples were then rinsed in PBS, blocked with $0.15 \%$ glycin in PBS, scraped in 1\% gelatin in PBS, pelleted, and embedded in $12 \%$ gelatin. Small blocks of pellet were cryoprotected with $2.3 \mathrm{M}$ sucrose, mounted on aluminum pins and frozen in liquid nitrogen. Ultrathin cryosections were cut at $-120^{\circ} \mathrm{C}$, placed on copper carrier grids, thawed and immunolabeled as previously described (48). In brief, the sections were incubated with blocking buffer containing fish skin gelatin (Sigma-Aldrich, G7765) and acetylated BSA (Aurion, 900.022) and immunolabeled with biotinylated goat antiGFP antibody (Rockland, 600-106-215) at 1:300, followed by rabbit anti-biotin antibody (Rockland, 100-4198) at 1:10000. Subsequently, the sections were incubated with Protein A-conjugated $10 \mathrm{~nm}$ gold particles (Cell Microscopy Core, University Medical Center Utrecht, the Netherlands), stained with uranyl acetate followed by a methylcellulose-uranyl acetate mixture, and examined in a JEOL JEM-1010 electron microscope. Quantification of the diameters of IRE1a subdomain tubes and ER cisterns was performed using Fiji software (47)).

\section{Subtomogram averaging}

In U2OS cells each tilt series was collected from $-60^{\circ}$ to $+60^{\circ}$ with an increment of $1^{\circ}$ in an automated fashion using SerialEM at 4-6 $\mu \mathrm{m}$ underfocus. The image pixel size used for subtomogram averaging was $6.52 \AA$ (binned by 2). Subsequent subtomogram averaging was performed by the EMAN2 tomography pipeline (49). Initially unbinned tilt-series were automatically aligned and reconstructed using EMAN2. In total, 3 cryo-tomograms were generated to provide a sufficient number of particles for further processing. Particles were picked using EMAN2 particle picking software using a box size of 56x56x56 pixels. Briefly model points were placed every $10 \mathrm{~nm}$ along the length (approximately one helical turn), of the oligomer present inside the membrane tube. Particles of various orientations were picked, including top-views and side-views. In total 653 model points were picked. CTF estimation and correction was performed by EMAN2. An initial model was then generated in $\mathrm{C} 1$ with all 653 particles in 5 iterations. The iteration 5 map was aligned to the symmetry axis and was used as an initial model for subtomogram refinement using C2 and D2 symmetry. The first iteration D2 map generated in subtomogram refinement was used as a reference for a sub-tilt refinement step using helical symmetry in 5 iterations. The helical symmetry parameters were as follows; $\mathrm{C}$ symmetry $=2$, rotation about the $\mathrm{Z}$ axis $=$ $45^{\circ}, \mathrm{nsym}=2.5$, and $\mathrm{tz}=5$. The map produced by iteration 5 includes $80 \%$ of the best aligned particles and is shown in figure S10. To focus on one strand of the helix, an automatic mask was generated to improve alignment in 5 iterations. The one-stranded map produced by iteration 5 includes $80 \%$ of the best aligned particle and is shown in figure 4 . The particles were split into two subsets and resolution is measured by the Fourier shell correlation of these two density maps. The correct hand of the final map was determined by EMAN2. The particles were mapped back into the cryotomogram by EMAN2 using a pKeep of 0.6. 


\section{Model prediction}

A schematic illustrating the model building process has been included in Fig. S11. SWISSMODEL was used to predict the human active form of IRE1 $\alpha$, based on the human sequence and the $S$. cerevisiae IRE1-LD crystal structure (PDB:2BE1) as a template. To prepare the template for SWISS-MODEL, the missing loops in the crystal structure were built using Modeller in Chimera (50).

\section{Dimer-dimer interface generation}

Two predicted model IRE1 $\alpha$ dimers were superimposed onto the $S$. cerevisiae IRE1-LD crystal structure dimer-dimer in Chimera. The S. cerevisiae IRE1-LD crystal structure dimerdimer was then omitted, leaving a gap between the two predicated human IRE1 $\alpha$ dimers. A new interface was modelled in Chimera by translating one dimer along one axis until the gap was filled. To accommodate the new dimer-dimer interface in the double-helical map, the angle between the two dimers was made more acute to approximately $45^{\circ}$ (the rise of the helix). The resulting dimer was then placed into the map using the fit-to-map function in Chimera. The remainder of the helix was then built whilst maintaining the same dimer-dimer interface throughout the helix.

\section{Quantifications}

IRE1 $\alpha$ subdomain tube diameters were measured by drawing lines between the center of each membrane density perpendicular to tube membranes. Regions less than $30 \mathrm{~nm}$ away from a junction are excluded. Tube diameters for 12 representative tomograms were quantified as 409 measurements. Distributions of immunogold particles shown in Fig. 2 were generated by manually extracting the $\mathrm{X}-\mathrm{Y}$ coordinates of each gold particle and using a python script to measure all pairwise distances and plotting the distance distribution. Line intensity plots shown in Fig. 3 were generated by averaging and aligning multiple line plots across each subdomain cross section. The resulting averaged plates were subsequently aligned and averaged as shown in Fig. 3C. Quantification of radii of curvature was performed by dividing IRE1 $\alpha$ subdomain tubes clearly visible in XY planes into tiling $25 \mathrm{~nm}$ segments, excluding junctions. Circles with diameters in increments of $50 \mathrm{~nm}$ were then manually fitted to each segment to yield estimates of the radius of curvature. $\mathrm{N}=274$.

\section{Calculations}

Calculations of IRE1 domain concentration assumes IRE1 $\alpha$ subdomain tubes to be perfect cylinders of $28.3 \mathrm{~nm}$ diameter measured from membrane centers with a membrane thickness of $37.5 \AA$ (51). Each $100 \mathrm{~nm}$ segment of such cylinder contains 139 IRE1-LD monomers, approximated from a pitch of $173 \AA$ with 24 monomers per turn. The volume occupied by IRE $1 \alpha-L D$ is calculated by using a glycosylated MW of $49196 \mathrm{~g} / \mathrm{mol}$ and a density of $1.35 \mathrm{~g} / \mathrm{mL}$ (52), yielding a volume of $8.41 \times 10^{3} \mathrm{~nm}^{3}$ occupied by 139 IRE1-LD monomers and $3.87 \times 10^{4} \mathrm{~nm}^{3}$ void volume. In this void volume, a single substrate molecule has a concentration of $43 \mu \mathrm{M}$. IRE1 density fold change upon stress are approximated from 139 monomer per $0.008890 \mu \mathrm{M}^{2}$ and contrasting to earlier calculations (25). IRE1LD concentration is extracted from the molarity of 139 units as $2.31 \times 10^{-22}$ mole / 4.7144* $10^{-20}$ L. IRE1 cytosolic domain concentrations are estimated from the approximate volume experienced by this domain $\left(1.05 \times 10^{6} \mathrm{~nm}^{3}\right.$ which assumes the domain extends $44 \mathrm{~nm}$ from the membrane due to a stretched cytosolic linker $+\mathrm{KR}$ domain height from crystal structures). The fold compaction for the KR domain from full distribution along cylindrical tubes to a flat ER membrane compares the experienced volume between a hollow cylinder as above and a column trapezoid with a volume of $2.49 \times 10^{5}$ $\mathrm{nm}^{3}$, which yielded the $\sim 4$ fold increase. 


\section{References}

1. A. Helenius, T. Marquardt, I. Braakman, The endoplasmic reticulum as a protein-folding compartment. Trends Cell Biol. 2, 227-231 (1992).

2. G. E. Karagöz, D. Acosta-Alvear, P. Walter, The Unfolded Protein Response: Detecting and Responding to Fluctuations in the Protein-Folding Capacity of the Endoplasmic Reticulum. Cold Spring Harb Perspect Biol. 11 (2019), doi:10.1101/cshperspect.a033886.

3. K. Mori, W. Ma, M. J. Gething, J. Sambrook, A transmembrane protein with a cdc2+/CDC28-related kinase activity is required for signaling from the ER to the nucleus. Cell. 74, 743-756 (1993).

4. J. S. Cox, C. E. Shamu, P. Walter, Transcriptional induction of genes encoding endoplasmic reticulum resident proteins requires a transmembrane protein kinase. Cell. $\mathbf{7 3}$, 1197-1206 (1993).

5. I. Tabas, D. Ron, Integrating the mechanisms of apoptosis induced by endoplasmic reticulum stress. Nat Cell Biol. 13, 184-190 (2011).

6. J. H. Lin, H. Li, D. Yasumura, H. R. Cohen, C. Zhang, B. Panning, K. M. Shokat, M. M. Lavail, P. Walter, IRE1 signaling affects cell fate during the unfolded protein response. Science. 318, 944-949 (2007).

7. M. Lu, D. A. Lawrence, S. Marsters, D. Acosta-Alvear, P. Kimmig, A. S. Mendez, A. W. Paton, J. C. Paton, P. Walter, A. Ashkenazi, Opposing unfolded-protein-response signals converge on death receptor 5 to control apoptosis. Science. 345, 98-101 (2014).

8. T.-K. Chang, D. A. Lawrence, M. Lu, J. Tan, J. M. Harnoss, S. A. Marsters, P. Liu, W. Sandoval, S. E. Martin, A. Ashkenazi, Coordination between Two Branches of the Unfolded Protein Response Determines Apoptotic Cell Fate. Mol Cell. 71, 629-636.e5 (2018).

9. C. Hetz, K. Zhang, R. J. Kaufman, Mechanisms, regulation and functions of the unfolded protein response. Nat Rev Mol Cell Biol. 21, 421-438 (2020).

10. X. Z. Wang, H. P. Harding, Y. Zhang, E. M. Jolicoeur, M. Kuroda, D. Ron, Cloning of mammalian Ire1 reveals diversity in the ER stress responses. EMBO J. 17, 5708-5717 (1998).

11. C. Sidrauski, P. Walter, The transmembrane kinase Ire $1 p$ is a site-specific endonuclease that initiates mRNA splicing in the unfolded protein response. Cell. 90, 1031-1039 (1997).

12. A. Bertolotti, Y. Zhang, L. M. Hendershot, H. P. Harding, D. Ron, Dynamic interaction of BiP and ER stress transducers in the unfolded-protein response. Nat Cell Biol. 2 , 326-332 (2000).

13. B. M. Gardner, P. Walter, Unfolded proteins are Ire1activating ligands that directly induce the unfolded protein response. Science. 333, 1891-1894 (2011).

14. G. E. Karagöz, D. Acosta-Alvear, H. T. Nguyen, C. P. Lee, F. Chu, P. Walter, An unfolded protein-induced conformational switch activates mammalian IRE1. Elife. 6 (2017), doi:10.7554/eLife.30700.

15. M. M. U. Ali, T. Bagratuni, E. L. Davenport, P. R. Nowak, M. C. Silva-Santisteban, A. Hardcastle, C. McAndrews, M. G. Rowlands, G. J. Morgan, W. Aherne, I. Collins, F. E. Davies, L. H. Pearl, Structure of the Ire1 autophosphorylation complex and implications for the unfolded protein response. EMBO J. 30, 894-905 (2011).

16. R. Volmer, K. van der Ploeg, D. Ron, Membrane lipid saturation activates endoplasmic reticulum unfolded protein response transducers through their transmembrane domains. Proc Natl Acad Sci US A. 110, 4628-4633 (2013).

17. K. Halbleib, K. Pesek, R. Covino, H. F. Hofbauer, D. Wunnicke, I. Hänelt, G. Hummer, R. Ernst, Activation of the Unfolded Protein Response by Lipid Bilayer Stress. Mol Cell. 67, 673-684.e8 (2017).

18. M. Calfon, H. Zeng, F. Urano, J. H. Till, S. R. Hubbard, H. P. Harding, S. G. Clark, D. Ron, IRE1 couples endoplasmic reticulum load to secretory capacity by processing the XBP-1 mRNA. Nature. 415, 92-96 (2002).

19. J. Hollien, J. S. Weissman, Decay of endoplasmic reticulum-localized mRNAs during the unfolded protein response. Science. 313, 104-107 (2006).

20. D. Bae, K. A. Moore, J. M. Mella, S. Y. Hayashi, J. Hollien, Degradation of Blos1 mRNA by IRE1 repositions lysosomes and protects cells from stress. J Cell Biol. 218, 1118-1127 (2019).

21. T. Aragón, E. van Anken, D. Pincus, I. M. Serafimova, A. V. Korennykh, C. A. Rubio, P. Walter, Messenger RNA targeting to endoplasmic reticulum stress signalling sites. Nature. 457, 736-740 (2009).

22. H. Li, A. V. Korennykh, S. L. Behrman, P. Walter, Mammalian endoplasmic reticulum stress sensor IRE1 signals by dynamic clustering. Proc Natl Acad Sci US A. 107, 1611316118 (2010).

23. J. J. Credle, J. S. Finer-Moore, F. R. Papa, R. M. Stroud, P. Walter, On the mechanism of sensing unfolded protein in the endoplasmic reticulum. Proc Natl Acad Sci US A. 102, 18773-18784 (2005).

24. J. Zhou, C. Y. Liu, S. H. Back, R. L. Clark, D. Peisach, Z. Xu, R. J. Kaufman, The crystal structure of human IRE1 luminal domain reveals a conserved dimerization interface required for activation of the unfolded protein response. Proc Natl Acad Sci U S A. 103, 14343-14348 (2006).

25. A. V. Korennykh, P. F. Egea, A. A. Korostelev, J. Finer-Moore, C. Zhang, K. M. Shokat, R. M. Stroud, P. Walter, The unfolded protein response signals through highorder assembly of Ire1. Nature. 457, 687-693 (2009).

26. D. Ricci, I. Marrocco, D. Blumenthal, M. Dibos, D. Eletto, J. Vargas, S. Boyle, Y. Iwamoto, S. Chomistek, J. C. Paton, A. W. Paton, Y. Argon, Clustering of IRE1 $\alpha$ depends on sensing ER stress but not on its RNase activity. The FASEB Journal. 33, 9811-9827 (2019).

27. V. Belyy, N.-H. Tran, P. Walter, Quantitative microscopy reveals dynamics and fate of clustered IRE1 $\alpha$. Proc Natl Acad Sci USA. 117, 1533-1542 (2020).

28. S. Asano, B. D. Engel, W. Baumeister, In Situ CryoElectron Tomography: A Post-Reductionist Approach to Structural Biology. J Mol Biol. 428, 332-343 (2016).

29. S. D. Carter, J. I. Mamede, T. J. Hope, G. J. Jensen, Correlated cryogenic fluorescence microscopy and electron cryo-tomography shows that exogenous TRIM5 $\alpha$ can form hexagonal lattices or autophagy aggregates in vivo. PNAS. 117, 29702-29711 (2020).

30. W. F. Tivol, A. Briegel, G. J. Jensen, An improved cryogen for plunge freezing. Microsc Microanal. 14, 375-379 (2008). 
31. A. Briegel, S. Chen, A. J. Koster, J. M. Plitzko, C. L. Schwartz, G. J. Jensen, Correlated light and electron cryomicroscopy. Meth. Enzymol. 481, 317-341 (2010).

32. S. D. Carter, S. K. Mageswaran, Z. J. Farino, J. I. Mamede, C. M. Oikonomou, T. J. Hope, Z. Freyberg, G. J. Jensen, Distinguishing signal from autofluorescence in cryogenic correlated light and electron microscopy of mammalian cells. J. Struct. Biol. 201, 15-25 (2018).

33. M. Chen, W. Dai, S. Y. Sun, D. Jonasch, C. Y. He, M. F. Schmid, W. Chiu, S. J. Ludtke, Convolutional neural networks for automated annotation of cellular cryo-electron tomograms. Nature Methods. 14, 983-985 (2017).

34. A. Waterhouse, M. Bertoni, S. Bienert, G. Studer, G. Tauriello, R. Gumienny, F. T. Heer, T. A. P. de Beer, C. Rempfer, L. Bordoli, R. Lepore, T. Schwede, SWISSMODEL: homology modelling of protein structures and complexes. Nucleic Acids Res. 46, W296-W303 (2018).

35. N. Borgese, M. Francolini, E. Snapp, Endoplasmic reticulum architecture: structures in flux. Curr Opin Cell Biol. 18, 358-364 (2006).

36. J. Nixon-Abell, C. J. Obara, A. V. Weigel, D. Li, W. R. Legant, C. S. Xu, H. A. Pasolli, K. Harvey, H. F. Hess, E. Betzig, C. Blackstone, J. Lippincott-Schwartz, Increased spatiotemporal resolution reveals highly dynamic dense tubular matrices in the peripheral ER. Science. 354, aaf3928aaf3928 (2016).

37. M. J. Phillips, G. K. Voeltz, Structure and function of ER membrane contact sites with other organelles. Nat Rev Mol Cell Biol. 17, 69-82 (2016).

38. S. Schuck, W. A. Prinz, K. S. Thorn, C. Voss, P. Walter, Membrane expansion alleviates endoplasmic reticulum stress independently of the unfolded protein response. J Cell Biol. 187, 525-536 (2009).

39. T. Cui-Wang, C. Hanus, T. Cui, T. Helton, J. Bourne, D. Watson, K. M. Harris, M. D. Ehlers, Local Zones of Endoplasmic Reticulum Complexity Confine Cargo in Neuronal Dendrites. Cell. 148, 309-321 (2012).

40. M. Sanches, N. M. Duffy, M. Talukdar, N. Thevakumaran, D. Chiovitti, M. D. Canny, K. Lee, I. Kurinov, D. Uehling, R. Al-awar, G. Poda, M. Prakesch, B. Wilson, V. Tam, C. Schweitzer, A. Toro, J. L. Lucas, D. Vuga, L. Lehmann, D. Durocher, Q. Zeng, J. B. Patterson, F. Sicheri, Structure and mechanism of action of the hydroxy-arylaldehyde class of IRE1 endoribonuclease inhibitors. Nat Commun. 5, 4202 (2014).

41. M. Puhka, M. Joensuu, H. Vihinen, I. Belevich, E. Jokitalo, Progressive sheet-to-tubule transformation is a general mechanism for endoplasmic reticulum partitioning in dividing mammalian cells. MBoC. 23, 2424-2432 (2012).

42. T. Shemesh, R. W. Klemm, F. B. Romano, S. Wang, J. Vaughan, X. Zhuang, H. Tukachinsky, M. M. Kozlov, T. A. Rapoport, A model for the generation and interconversion of ER morphologies. Proc Natl Acad Sci USA. 111, E5243E5251 (2014).

43. S. Q. Zheng, B. Keszthelyi, E. Branlund, J. M. Lyle, M. B. Braunfeld, J. W. Sedat, D. A. Agard, UCSF tomography: an integrated software suite for real-time electron microscopic tomographic data collection, alignment, and reconstruction. $J$ Struct Biol. 157, 138-147 (2007).

44. D. N. Mastronarde, Automated electron microscope tomography using robust prediction of specimen movements. J Struct Biol. 152, 36-51 (2005).

45. J. R. Kremer, D. N. Mastronarde, J. R. McIntosh, Computer visualization of three-dimensional image data using IMOD. J. Struct. Biol. 116, 71-76 (1996).

46. J. I. Agulleiro, J. J. Fernandez, Fast tomographic reconstruction on multicore computers. Bioinformatics. 27, 582-583 (2011).

47. J. Schindelin, I. Arganda-Carreras, E. Frise, V. Kaynig, M. Longair, T. Pietzsch, S. Preibisch, C. Rueden, S. Saalfeld, B. Schmid, J.-Y. Tinevez, D. J. White, V. Hartenstein, K. Eliceiri, P. Tomancak, A. Cardona, Fiji: an open-source platform for biological-image analysis. Nature Methods. 9 , 676-682 (2012).

48. J. W. Slot, H. J. Geuze, Cryosectioning and immunolabeling. Nat Protoc. 2, 2480-2491 (2007).

49. M. Chen, J. M. Bell, X. Shi, S. Y. Sun, Z. Wang, S. J. Ludtke, A complete data processing workflow for cryo-ET and subtomogram averaging. Nature Methods. 16, 1161-1168 (2019).

50. E. F. Pettersen, T. D. Goddard, C. C. Huang, G. S. Couch, D. M. Greenblatt, E. C. Meng, T. E. Ferrin, UCSF Chimera--a visualization system for exploratory research and analysis. J Comput Chem. 25, 1605-1612 (2004).

51. K. Mitra, I. Ubarretxena-Belandia, T. Taguchi, G. Warren, D. M. Engelman, Modulation of the bilayer thickness of exocytic pathway membranes by membrane proteins rather than cholesterol. Proc Natl Acad Sci U S A. 101, 4083-4088 (2004).

52. H. Fischer, I. Polikarpov, A. F. Craievich, Average protein density is a molecular-weight-dependent function. Protein Sci. 13, 2825-2828 (2004). 


\section{Supplementary Materials}

Figs. S1 to S16

Captions for Movies S1 to S3

Other Supplementary Materials for this manuscript:

Movies S1 to S3 

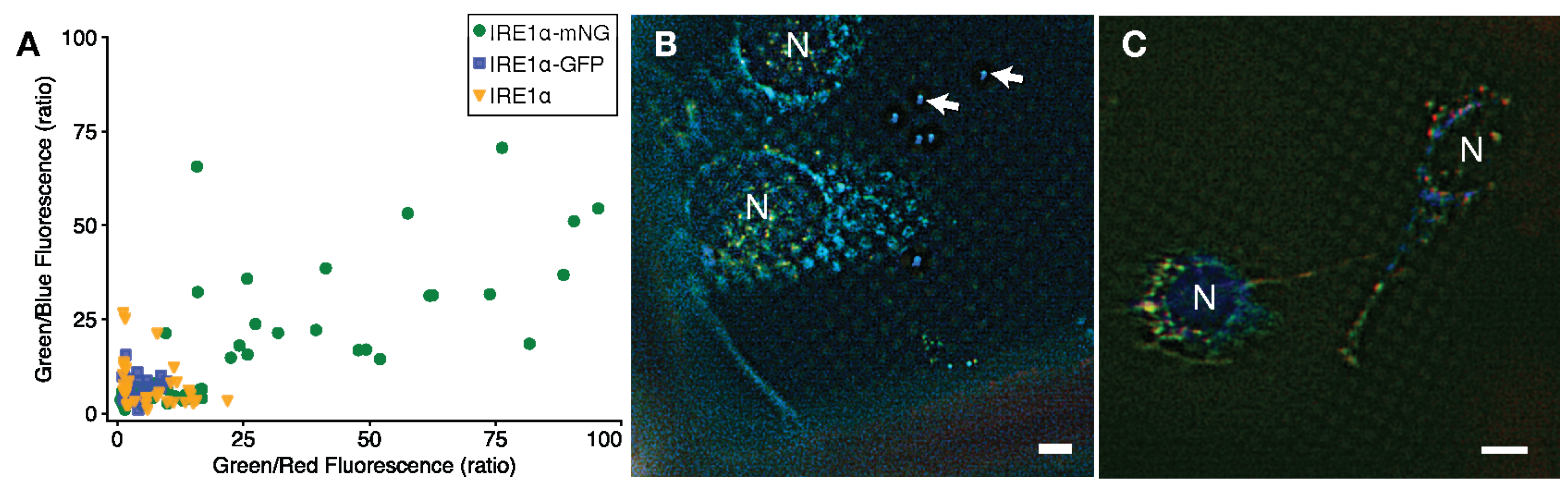

Fig. S1. Fluorescent profile across cell lines expressing different IRE1 $\alpha$ constructs.

(A) Scatter plot of fluorescent profiles for bright spots observed at liquid nitrogen temperature for stressed cells expressing IRE1 $\alpha-\mathrm{mNG}$, IRE1 $\alpha$-GFP or WT IRE1 $\alpha$. The ratios of Green/Red and Green/Blue fluorescence intensity taken at the same exposures across channels are used to identify specific IRE1 $\alpha$ signal. Representative cryo-fluorescent light microscope images of stressed MEFsIRE1 $\alpha$-GFP (B) and MEFs-IRE1 $\alpha$ (C; lacking fluorescent protein) cells grown directly on coated Quantifoil grids imaged at 1 second exposure in BFP, GFP and mCherry channels. Cells were treated with Tm for 2 hours prior to cryo-preservation and imaged in liquid nitrogen. Nuclei are indicated with "N." White arrows: $500 \mathrm{~nm}$ fluorescent nanosphere. Scale bars are $6 \mu \mathrm{m}$. 

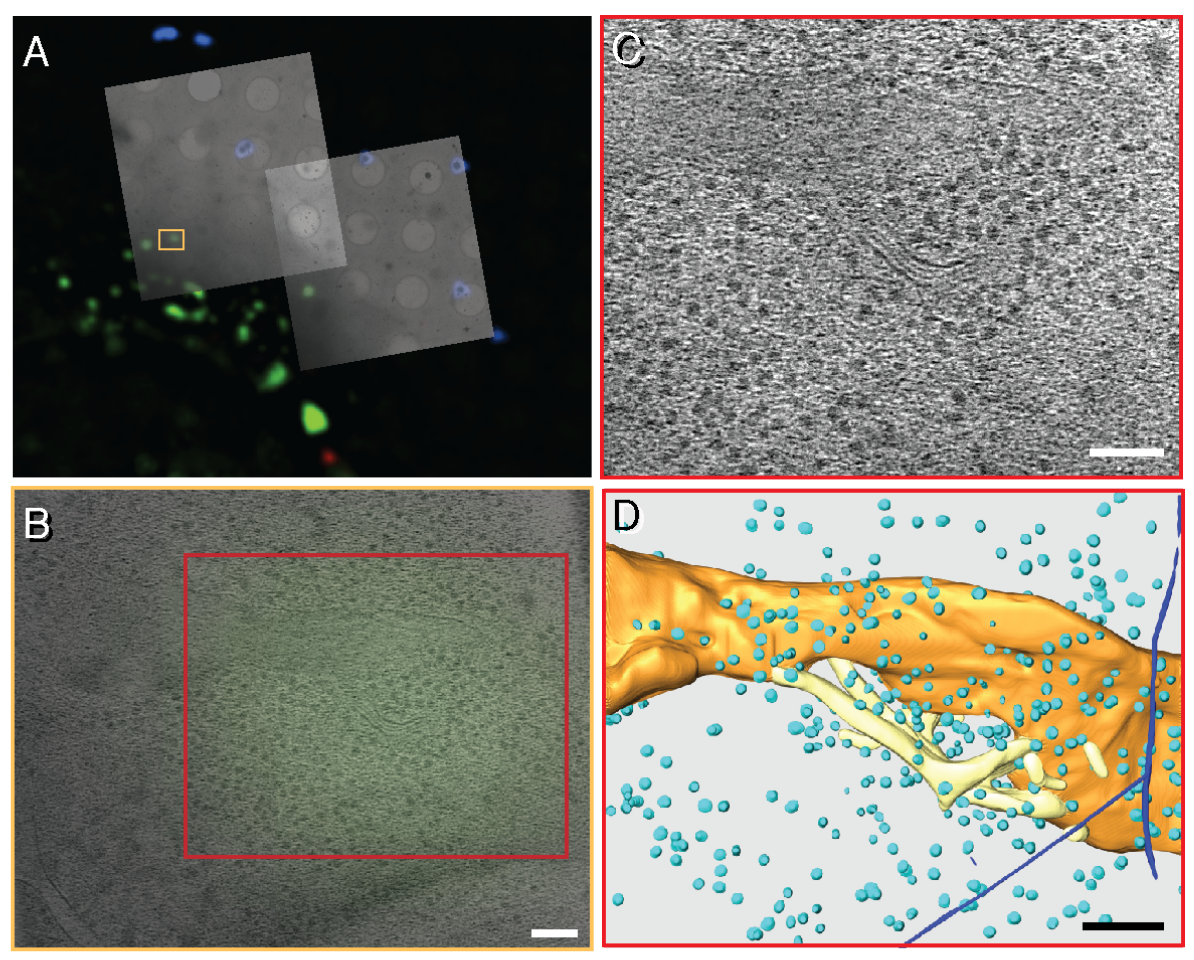

Fig. S2. Target identification by correlation and tomographic reconstruction in intact MEFs cells expressing IRE1 $\alpha$-mNeonGreen.

(A) Overlay of multi-channel cryo-LM images with low magnification (3000X) cryo electron micrographs of regions containing IRE1-mNeonGreen foci formed after 2 hours ER stress induction. Blue fluorescent spheres visible by both light and electron microscopy were used to align the images in $\mathrm{X}$ and $\mathrm{Y}$ directions prior to targeting cell regions for high magnification tilt series. (B) Correlation of reconstructed tomogram with fluorescent signal reveals sub-regions with IRE1 foci in an additional example from the same cell depicted in Fig. 1B-F. (C) A z slice showing thin membrane tubes within regions colocalizing with IRE1-mNeonGreen signal. (D) manual 3D segmentation for tomogram in C. Orange: normal ER membrane. Yellow: thin ER membranes at IRE1-mNeonGreen regions. Teal: ribosomes. Blue: cytoskeletal elements. Scale bars: $100 \mathrm{~nm}$. 

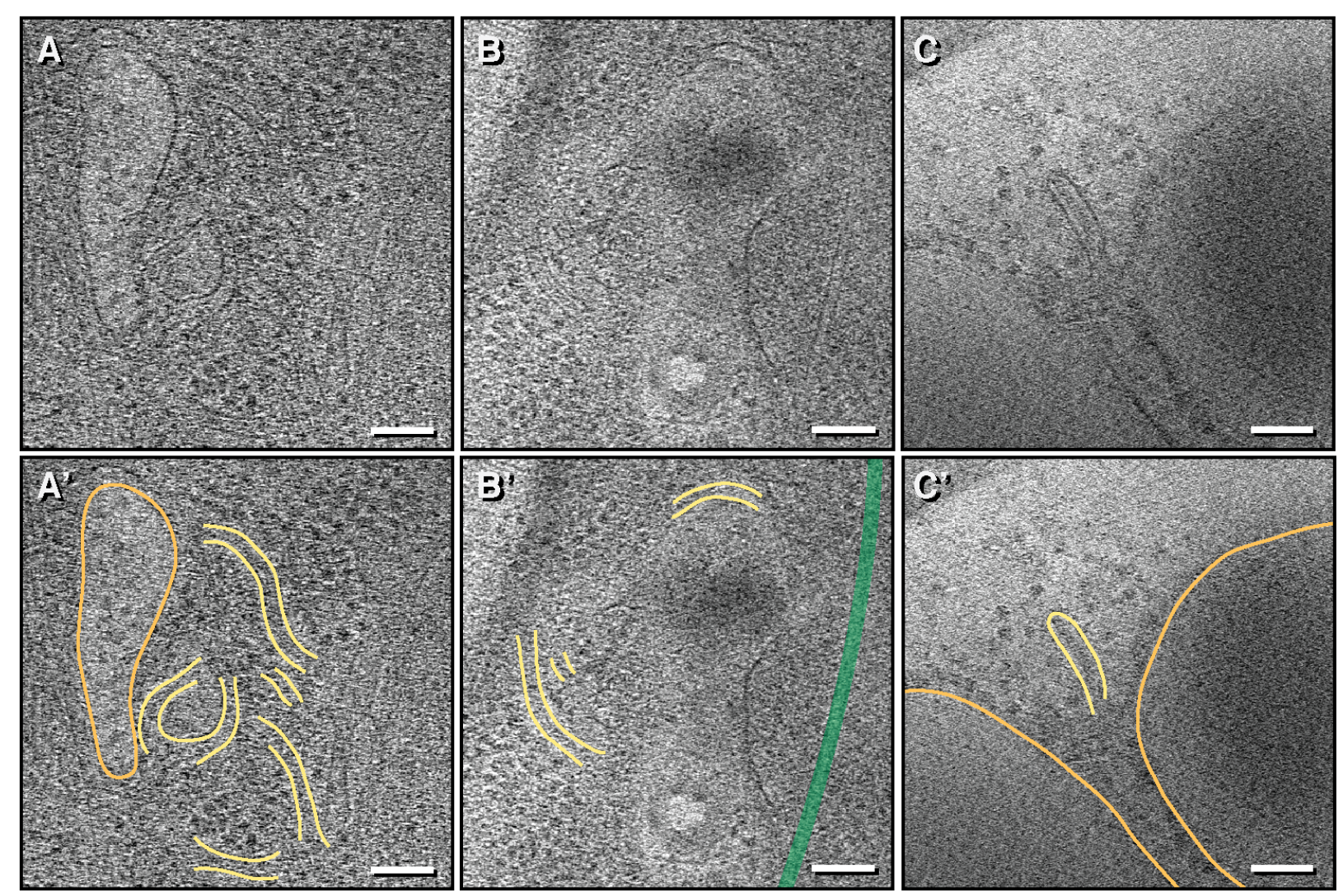

Fig. S3. Additional example of IRE1 $\alpha-\mathrm{mNG}$ fluorescence correlated to narrow membrane tubes in stressed MEFs-IRE1 $\alpha-\mathrm{mNG}$ cells.

(A-C) Representative $z$ slice of corresponding sub-regions correlated with IRE $1 \alpha-m N G$ signal in 3 different examples obtained in stressed cells expressing IRE1 $\alpha-m N G$. Scale bar $=100 \mathrm{~nm}$. (A'C') Segmentation of membranes and structures observed in tomograms where yellow $=$ thin ER tubes of IRE1 $\alpha$ subdomains, orange $=$ membrane of ER sheet/tubules, and green $=$ cytoskeletal components. 

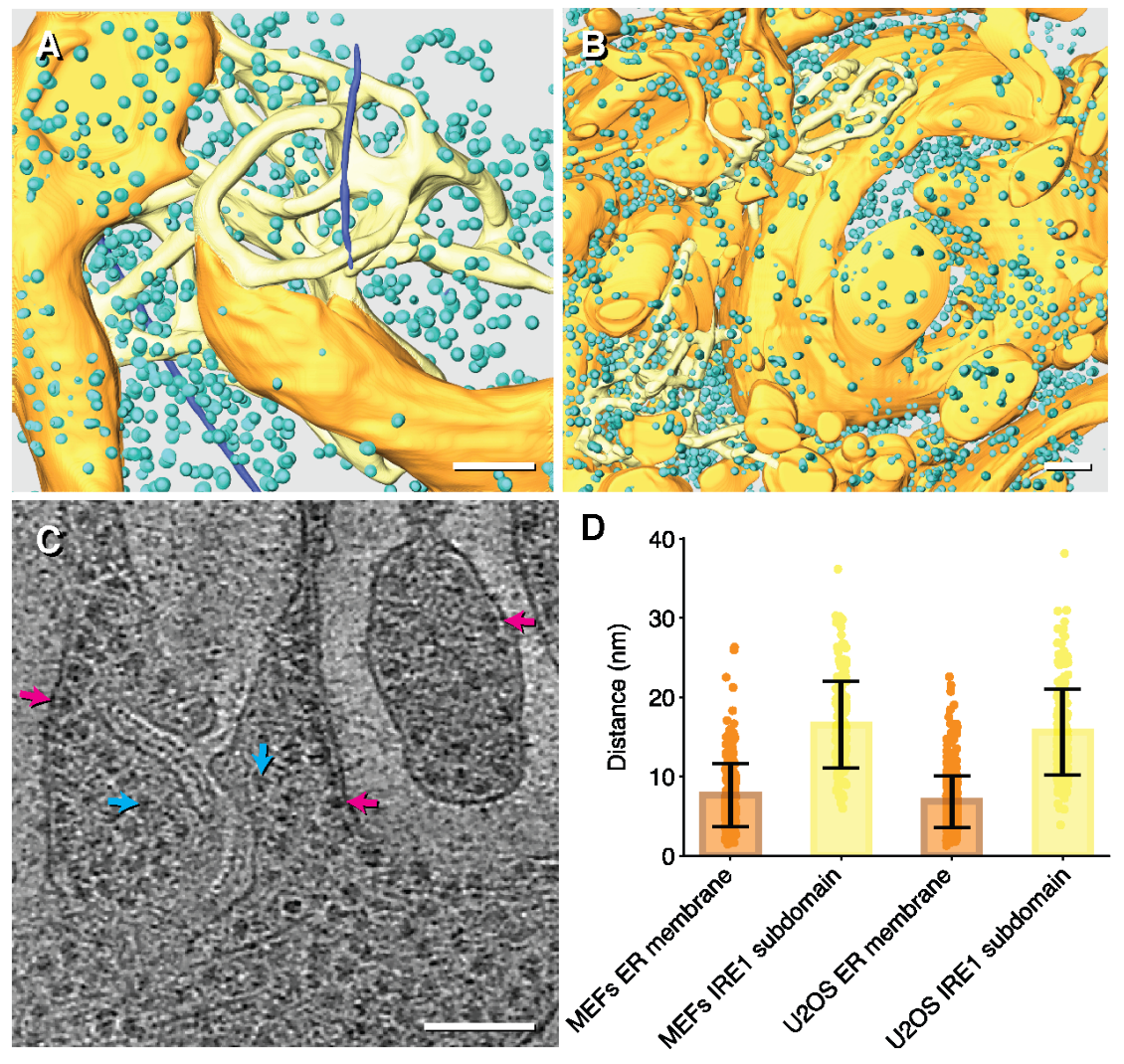

Fig. S4. IRE1 $\alpha$ subdomain tubes are devoid of bound ribosomes.

(A) Segmentation of MEFs tomogram corresponding to Fig. 1D but showing ribosomes and cytoskeletal segmentation. Scale bar $=100 \mathrm{~nm}$ (B) Segmentation of U2OS tomogram with larger cell region corresponding to Fig $1 \mathrm{~F}$ with ribosomes and cytoskeletal components shown. Scale bar $=100 \mathrm{~nm}$. (C) An example z slice of IRE1 $\alpha$ subdomain tube and adjacent ER sheet/tubules. Cyan arrows point to ribosomes near IRE1 $\alpha$ subdomains; Magenta arrows indicate ribosomes bound to ER membranes. Scale bar $=100 \mathrm{~nm}$. (D) Plot of distance between ribosomes and membranes comparing ER sheet/tubules membranes and IRE1 $\alpha$ subdomain membranes for tomograms in A and B. Error bar is standard deviation. $\mathrm{N}=218,180,215,519$. 

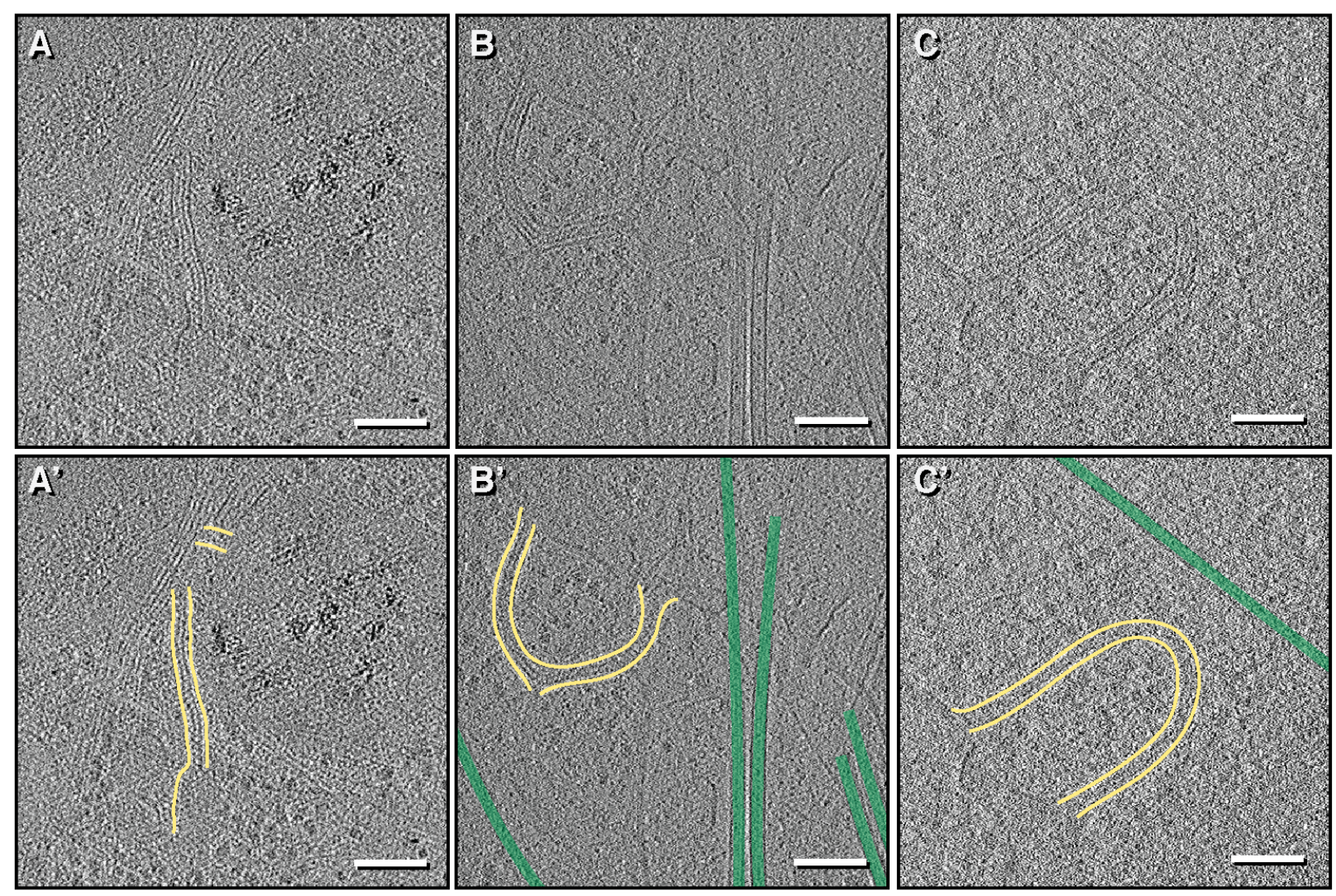

Fig. S5. Additional example of IRE1 $\alpha$ fluorescence correlated to narrow membrane tubes in stressed U2OS cells expressing fluorescently tagged IRE1 $\alpha$.

(A-B) Representative $z$ slice from two additional examples obtained in stressed U2OS-IRE1 $\alpha$ $\mathrm{mNG}$ cells expressing fluorescently tagged IRE1 $\alpha$. (C) representative $\mathrm{Z}$ slice from an example obtained from U2OS-IRE1 $\alpha$-mRuby cell line where IRE1 $\alpha$ is tagged at same location with an mRuby 3 red fluorescent protein instead of $m$ NeonGreen. Observation of narrow membrane tubes with specific red fluorescence using mRuby 3 supports that bright fluorescent foci are indeed IRE1 $\alpha$ foci rather than autofluorescent signal. (A'-C') Segmentation of membranes and structures observed in tomograms where yellow $=$ thin ER tubes of IRE1 $\alpha$ subdomains and green $=$ microtubules. Scale bar $=100 \mathrm{~nm}$. 


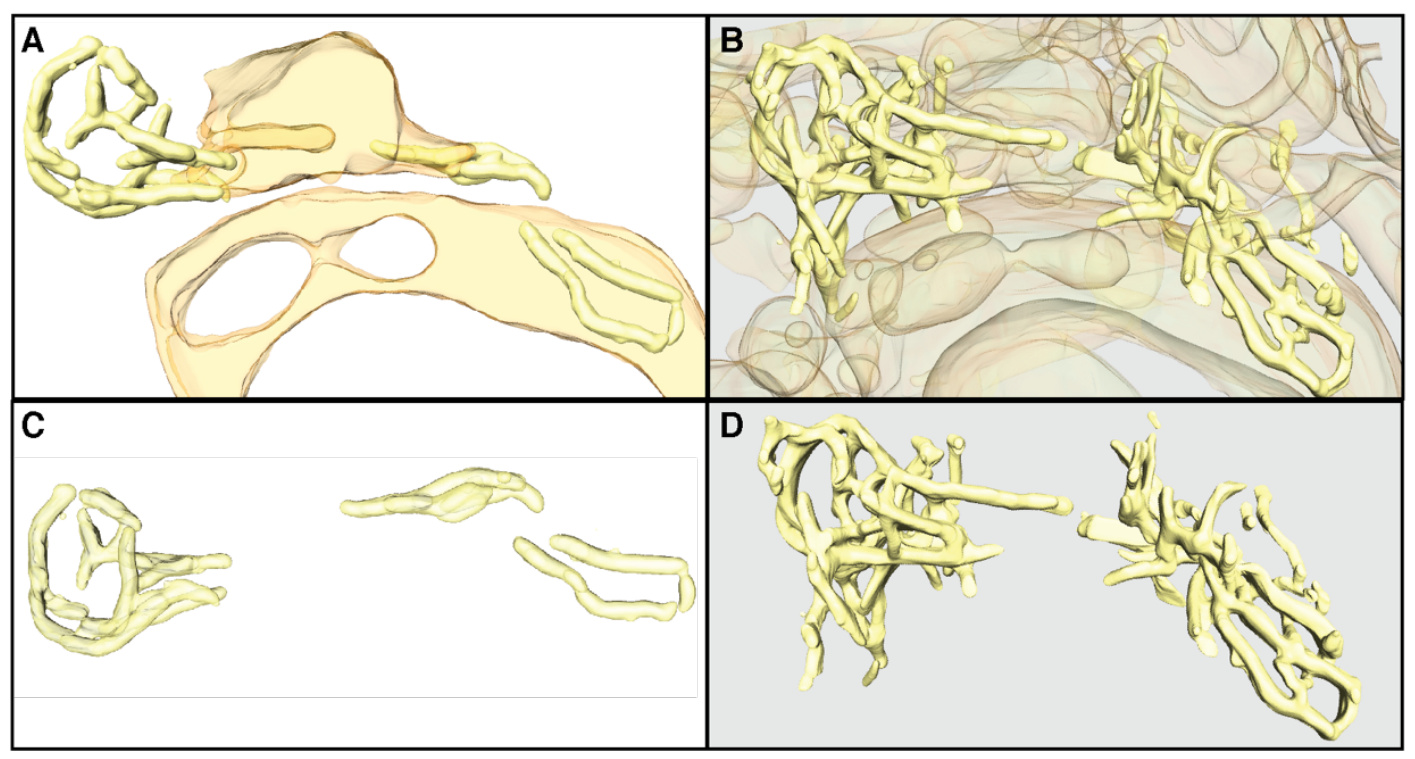

Fig. S6. Comparison of machine-learning and manual segmentation of U2OS tomogram.

(A) and (C) are isosurfaces generated from EMAN2 CNN segmentation where general ER structures are shown in (A) and only the narrow RE1 $\alpha$ subdomain tubes are shown in C. (B) and (D) are comparable views with smoothed isosurfaces generated manually using manual segmentation tools in Amira. A lot more structures are recognized by human eyes than are annotated by the neural network, especially at junctional regions. The manual segmentation in (B) and (D) therefore has higher degree of connectivity compared to (A) and (C). 

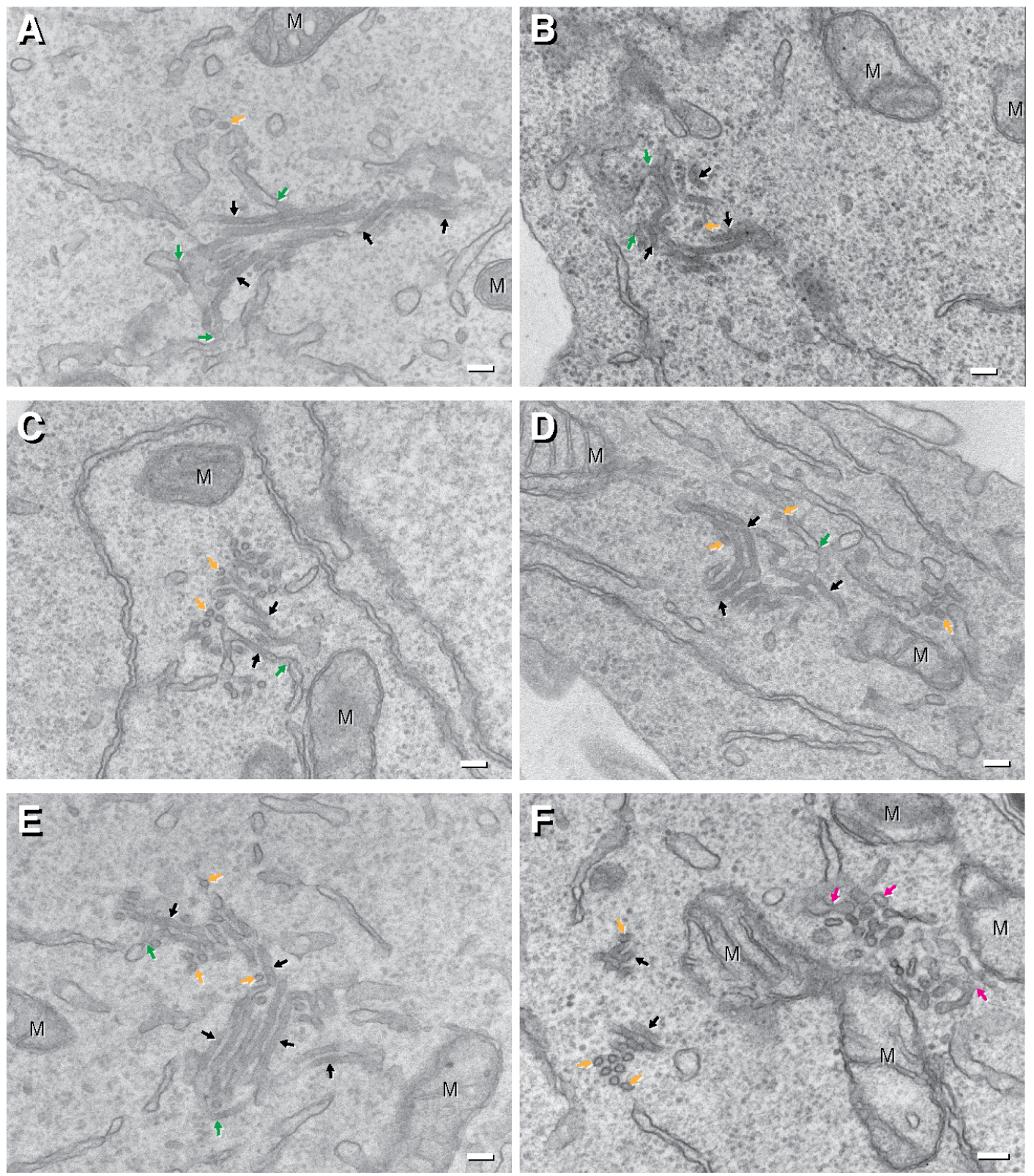

Fig. S7. Rare instances of IRE1 $\alpha$ subdomain can be observed in stressed cells expressing inducible IRE1 $\alpha$-GFP by conventional EM.

(A-F) Micrographs of thin sections of Epon-embedded cells. Thin membrane structures with diameters of $30 \pm 3 \mathrm{~nm}$ (error: standard deviation. $\mathrm{N}=89$ ) can be observed as rectangular longitudinal (black arrows) and circular end-on cross-sections (orange arrows). These narrow membranes are connected to larger ER structures (diameters of $53 \pm 20 \mathrm{~nm}, \mathrm{~N}=90$ ) at junctions indicated by green arrows. Intriguingly, the lumens of these structures appear darker than surrounding ER lumen, indicative of higher protein density. Scale bar $=100 \mathrm{~nm}$. $\mathrm{M}=$ mitochondria. These extremely narrow membrane tubes are morphologically distinct from ER exit sites (magenta arrows in F), which are finger-like protrusions with diameters of $35 \pm 7 \mathrm{~nm}(\mathrm{~N}=20)$ consistent with ER-derived vesicles. 

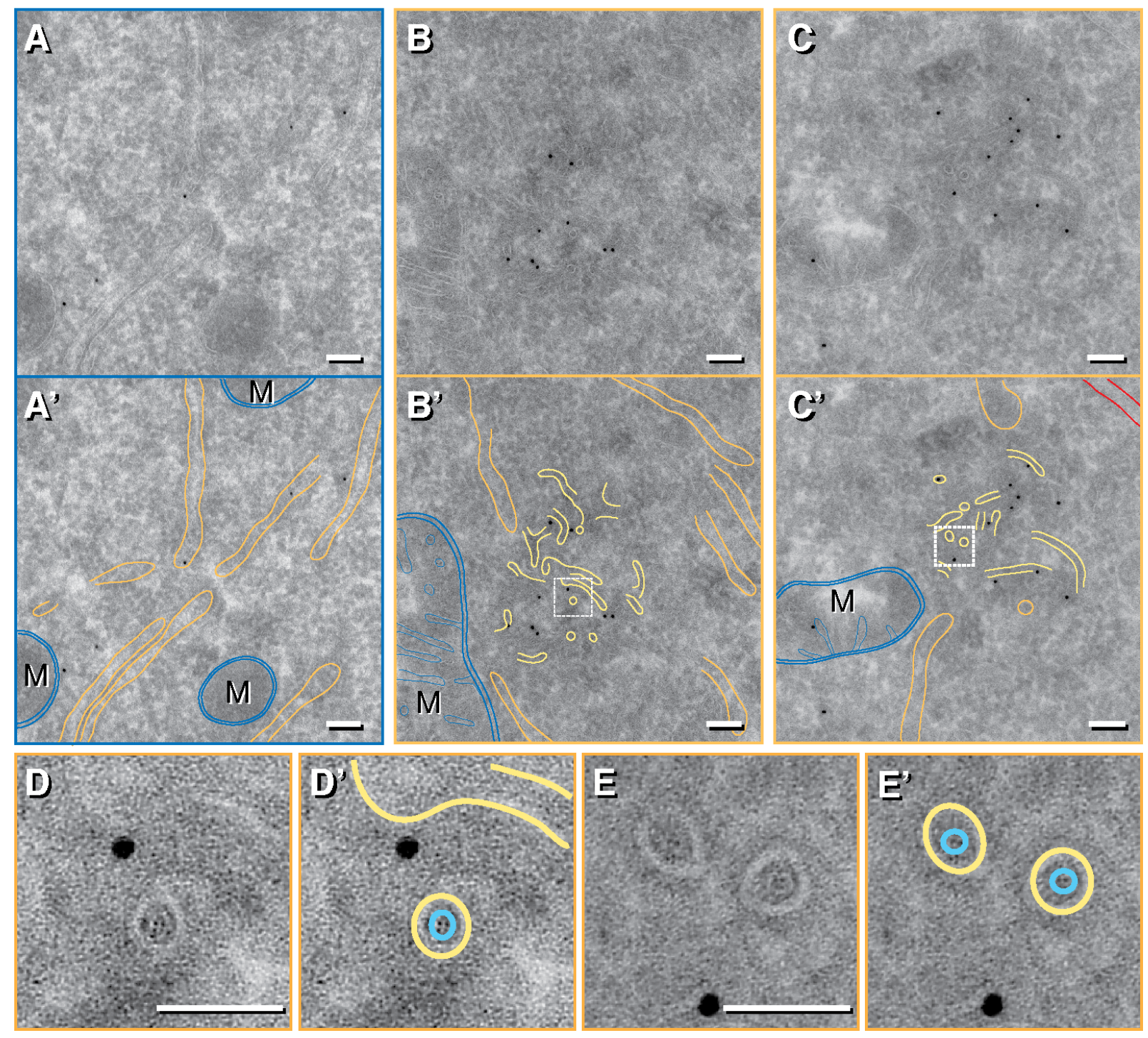

Fig. S8. Additional example of visualization of IRE1 $\alpha$ subdomain by immunogold EM.

(A, A') Micrograph of cell expressing IRE1 $\alpha$-GFP epitope but not stressed, showing disperse and sparse immunogold particles labeling general ER tubules/sheets segmented in orange. $\mathrm{M}=$ mitochondria are segmented in blue. Scale bars $=100 \mathrm{~nm}$. (B, B', C, C') Micrograph of immunogold-labeled stressed cells expressing IRE1 $\alpha$-GFP epitope. Large clusters of gold particles localize to regions with narrow membrane structures (segmented in yellow). These narrow tubes have an averaged diameter of $26 \pm 2 \mathrm{~nm}$ (error: standard deviation; $\mathrm{N}=108$ ) compared to surrounding ER structures segmented in orange with an averaged diameter of $51 \pm 18 \mathrm{~nm}$ (error: standard deviation; $\mathrm{N}=133$ ). $\mathrm{M}=$ mitochondria are segmented in blue. Plasma membranes seen in $\mathrm{C}$ are segmented in red. Scale bars $=100 \mathrm{~nm}$. Regions within white squares in B' and C' are enlarged in D and E, respectively. End-on cross sections of thin IRE1 $\alpha$ subdomain tubes appear as two roughly concentric rings, consistent with helical protein density within the lumen (cyan density). Scale bar $=50 \mathrm{~nm}$. 
bioRxiv preprint doi: https://doi.org/10.1101/2021.02.24.432779; this version posted February 24, 2021. The copyright holder for this preprint (which was not certified by peer review) is the author/funder, who has granted bioRxiv a license to display the preprint in perpetuity. It is made available under aCC-BY 4.0 International license.
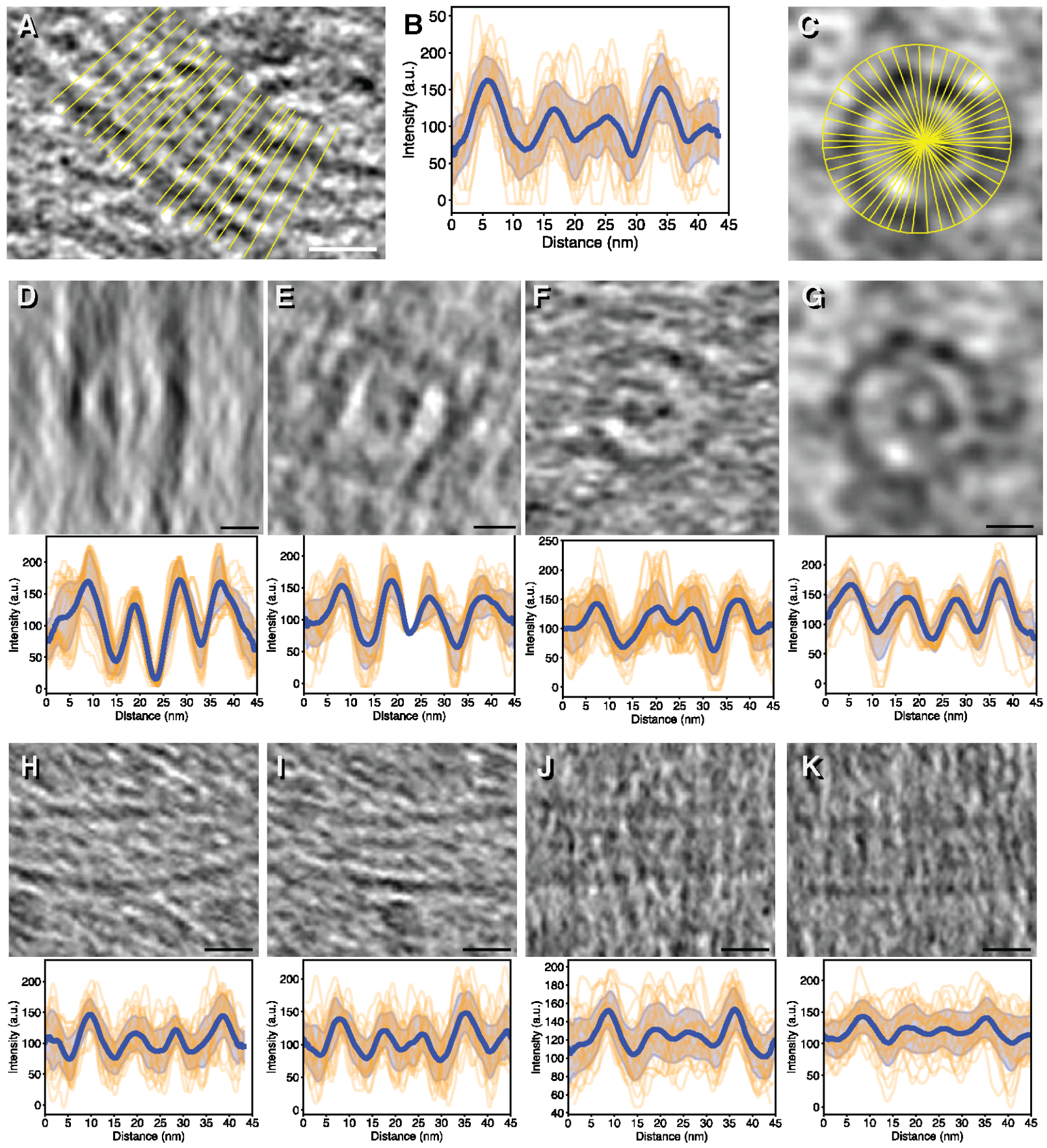

Fig. S9. IRE1 $\alpha$ subdomain tubes in MEFs cells contain lumenal protein density.

(A) Example of how line profiles were drawn for IRE1 $\alpha$ subdomain longitudinal cross sections and resulting averaged line plot (B) showing four distinct peaks. (C) Example of how line profiles were drawn for IRE1 $\alpha$ subdomain end-on cross section depicted in Fig. 3B. (D-K) IRE1 $\alpha$ subdomain cross sections and resulting averaged line plots used to generate plot of averaged profiles and measure diameters shown in Fig. 3C. Scale bars $=20 \mathrm{~nm}$. 


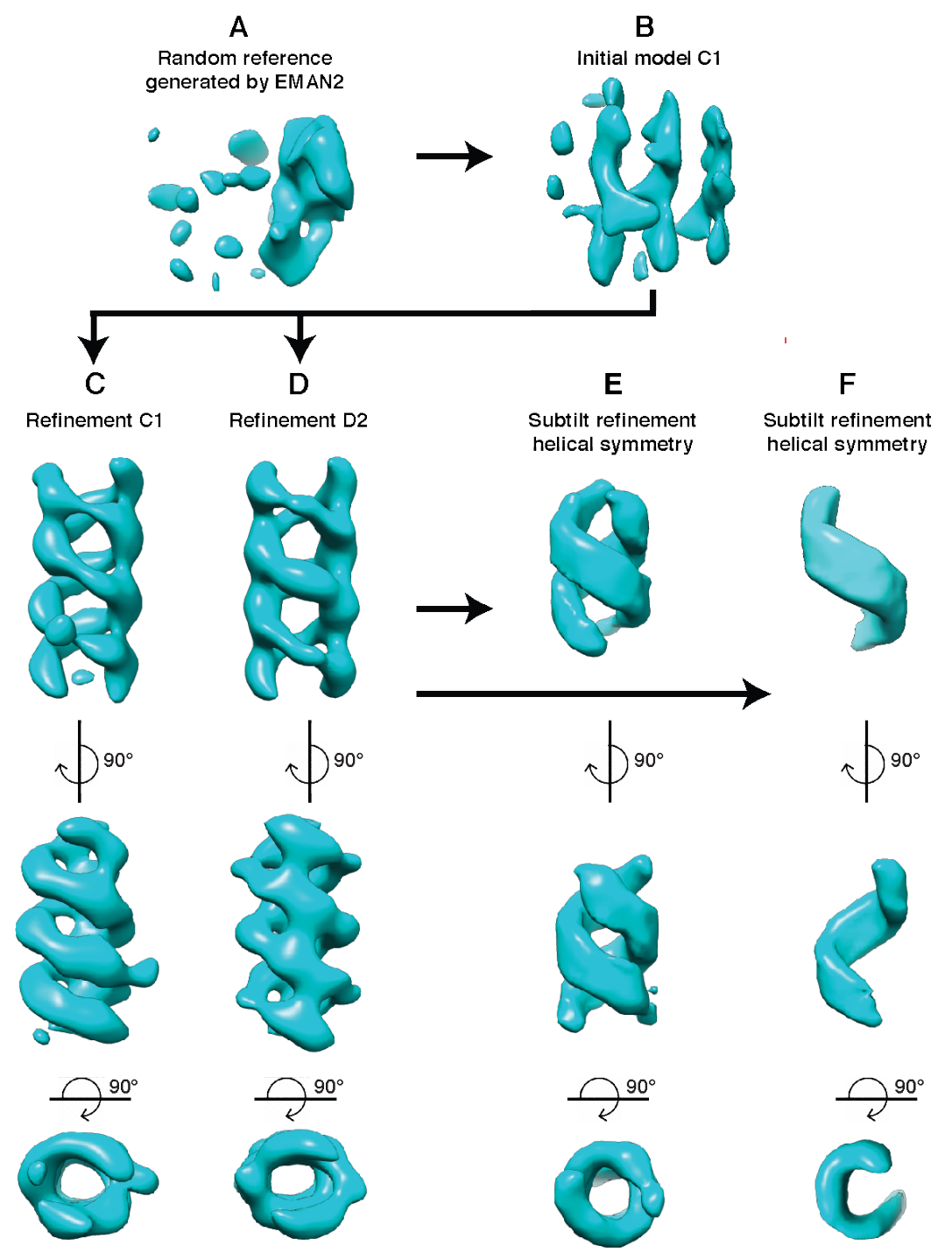

Fig. S10. Subtomogram average workflow.

(A) EMAN2 generated random reference. (B) Initial C1 model. (C) Refinement of the initial model in C1. (D) Refinement of the initial model in D2. This D2 average was used as a reference for all the following subtilt refinements, (E) helical symmetry (F) helical symmetry focused on one strand of the double-helical filament. 

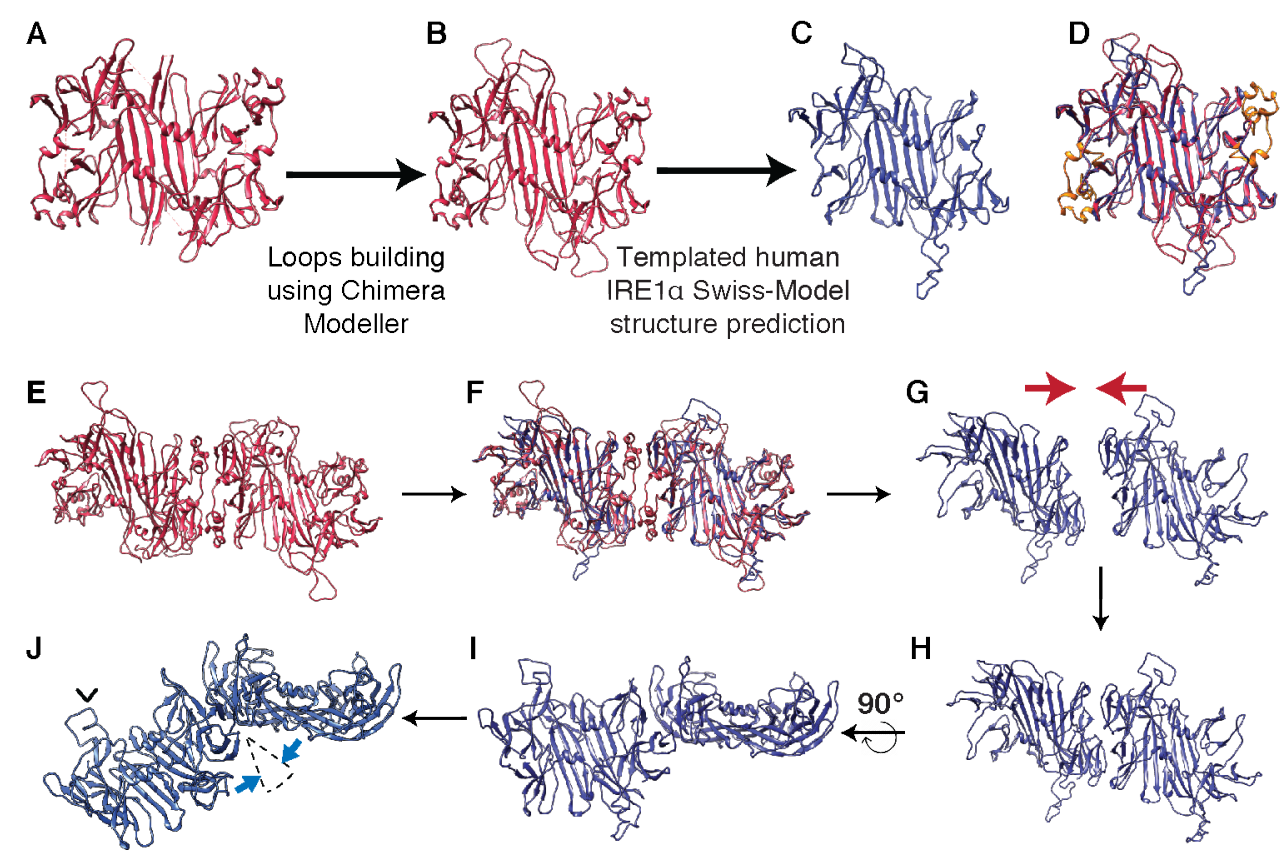

Fig. S11. Generation of a modeled human active IRE1 $\alpha$-LD and dimer-dimer interface.

(A-D) SWISS-MODEL homology modeling of the active human IRE1 $\alpha$ LD using the $S$. cerevisiae IRE1 lumenal crystal structure (PDB ID: 2BE1) as a template. (A) 2BE1. (B) 2BE1 with loops built using Modeller in Chimera. (C) Human IRE1 $\alpha$ lumenal structure predicted using the template generated in B. (D) Superimposing of 2BE1 (B; with loops) and modeled active human IRE1 $\alpha$ lumenal domain (C) reveals a helical bundle present in B but not in $\mathrm{C}$, shown in orange. (E) The S. cerevisiae IRE1 lumenal crystal structure (2BE1) dimer. (F) Two modeled active human IRE1 $\alpha$ LDs were superimposed onto the dimer in E. (G) Same superimposed human IRE1 $\alpha$-LDs as F, with the $S$. cerevisiae dimer removed. Red arrows highlight translational shift imposed on the two monomers to eliminate gap, resulting in a new interface shown in $\mathrm{H}$. (I) Same as $\mathrm{H}$, rotated $90^{\circ}$ around the axis of the interface hinge. $(\mathrm{J})$ Hinge angle decreased as indicated by blue arrows to accommodate the curvature of the sub-tomogram average maps. 
bioRxiv preprint doi: https://doi.org/10.1101/2021.02 24.432779. this version posted February 24, 2021. The copyright holder for this preprint (which was not certified by peer review) is the author/funder, who has granted bioRxiv a license to display the preprint in perpetuity. It is made available under aCC-BY 4.0 International license.

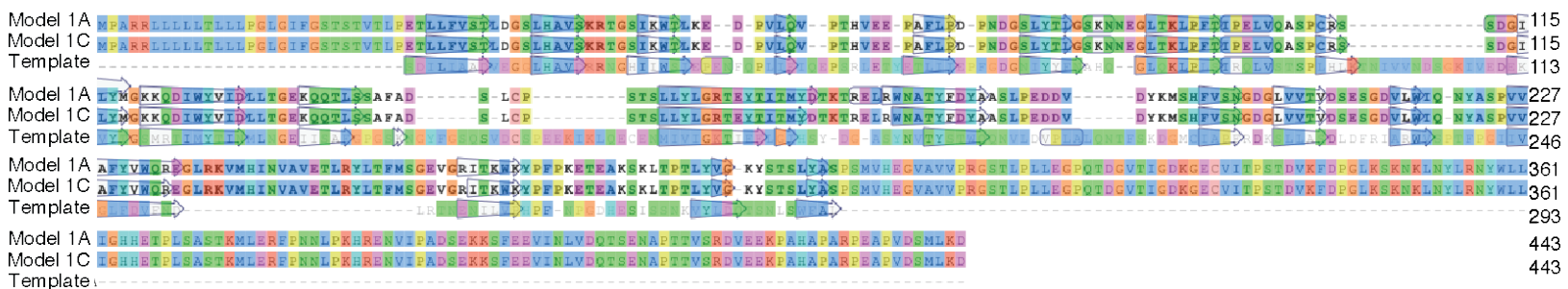

Fig. S12. Model-Template sequence and secondary structure alignment in DSSP format for the SWISS-MODEL homology modeling of the active human IRE1 $\alpha$ LD shown in S11. 
A
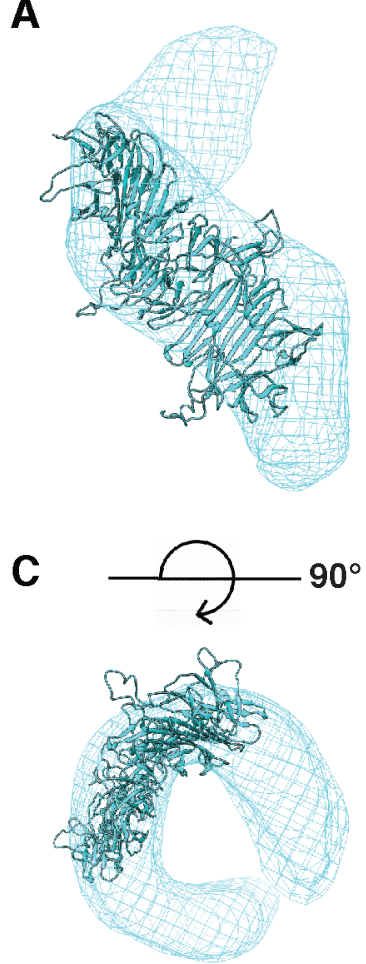

B

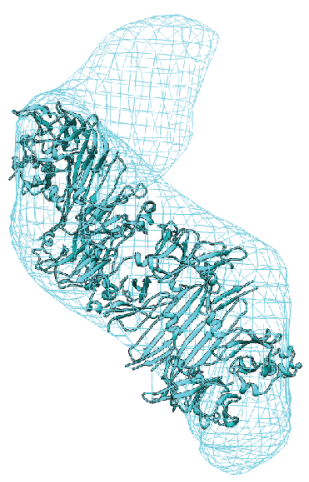

D
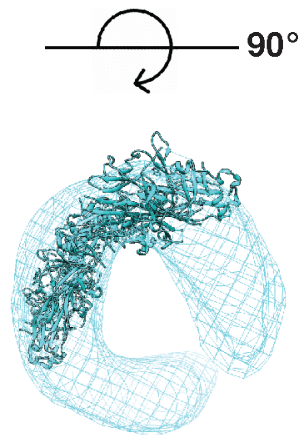

Fig. S13. Steps used to build double-helical model.

(A) The dimer-dimer in the human active IRE1 $\alpha$-LD fitted into map as a rigid body using fit in map function in Chimera. (B) Generation of an altered dimer-dimer interface based on the rise and twist of the helix was fitted into map as a rigid body using fit in map function in Chimera. (C, D) Same views as A and B, respectively, rotated around the $\mathrm{x}$-axis by $90^{\circ}$. 


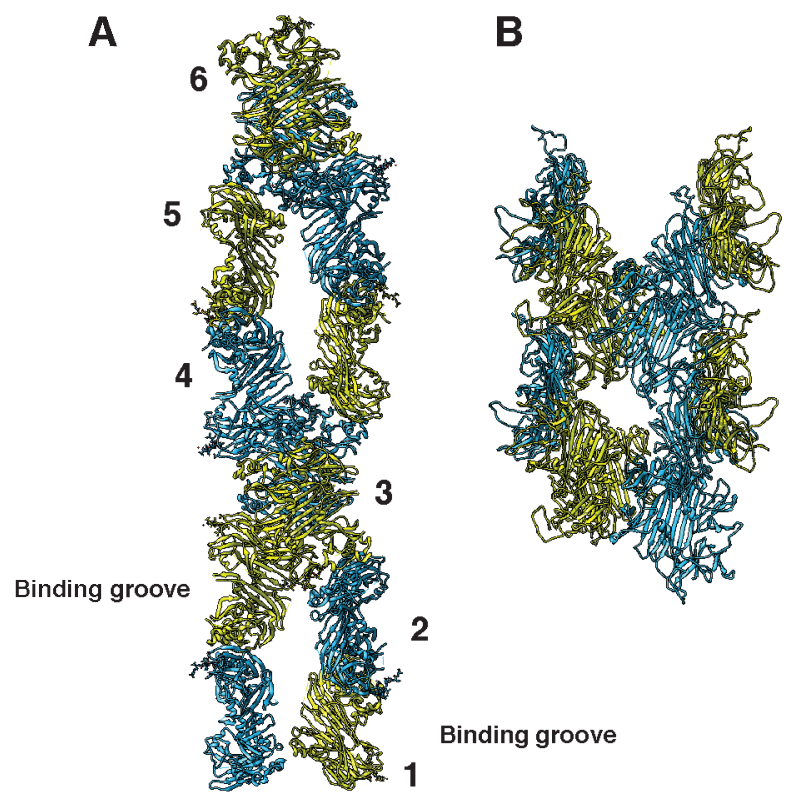

Fig. S14. Comparison of $S$. cerevisiae IRE1-LD 2BE1 and modeled active human IRE1 $\alpha$-LD oligomers.

(A) Double helical oligomer found in the crystal structure of the $S$. cerevisiae IRE1-LD with 12 dimers per double-helical turn and the predicted unfolded protein binding groove facing outward. Numbers indicate individual dimers running along one helical filament. (B) the human IRE1 $\alpha$-LD double helix modelled and fitted to the sub-tomogram averaging map. 


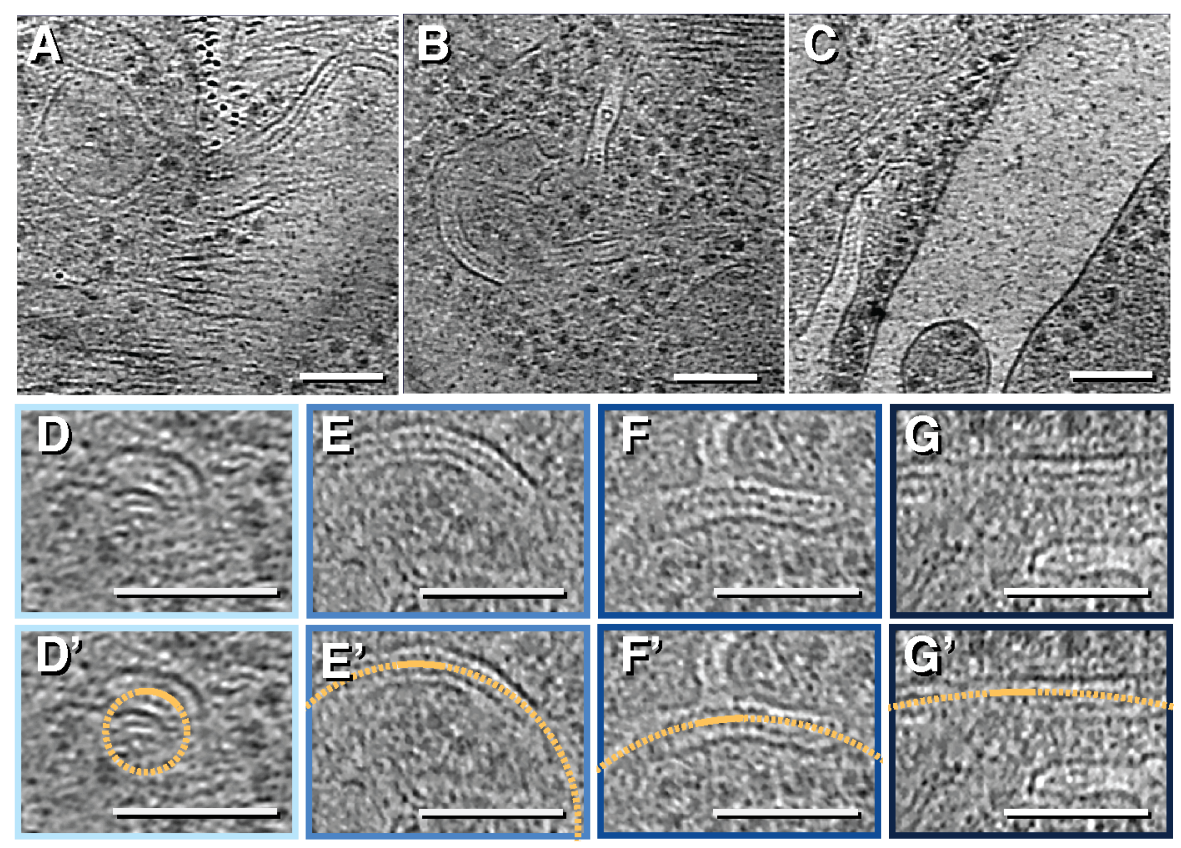

Fig. S15. Example range of curvatures observed for IRE1 $\alpha$ subdomains.

(A-C) Example regions from U2OS-IRE1-mNG cells showing IRE1 $\alpha$ subdomain tubes with varying degrees of curvature. Cropped regions exhibiting radii of curvature (ROC) ranging from 25-75 nm (D), 75-125 nm (E), 125-175 nm (F), and >175 nm (G) quantified and plotted in Fig. 4D. The outline color matches the bars in Fig. 4D, organized as increasing darkness corresponding to increasing ROC. A sample local curvature fit circle (dash orange circle) with ROC of $25 \mathrm{~nm}$ (D'), $100 \mathrm{~nm}\left(\mathrm{E}^{\prime}\right), 150 \mathrm{~nm}\left(\mathrm{~F}^{\prime}\right)$ and $400 \mathrm{~nm}\left(\mathrm{G}^{\prime}\right)$ are shown for representative $25 \mathrm{~nm}$ segments (solid orange arcs) within the view shown. All scale bars $=100 \mathrm{~nm}$. 


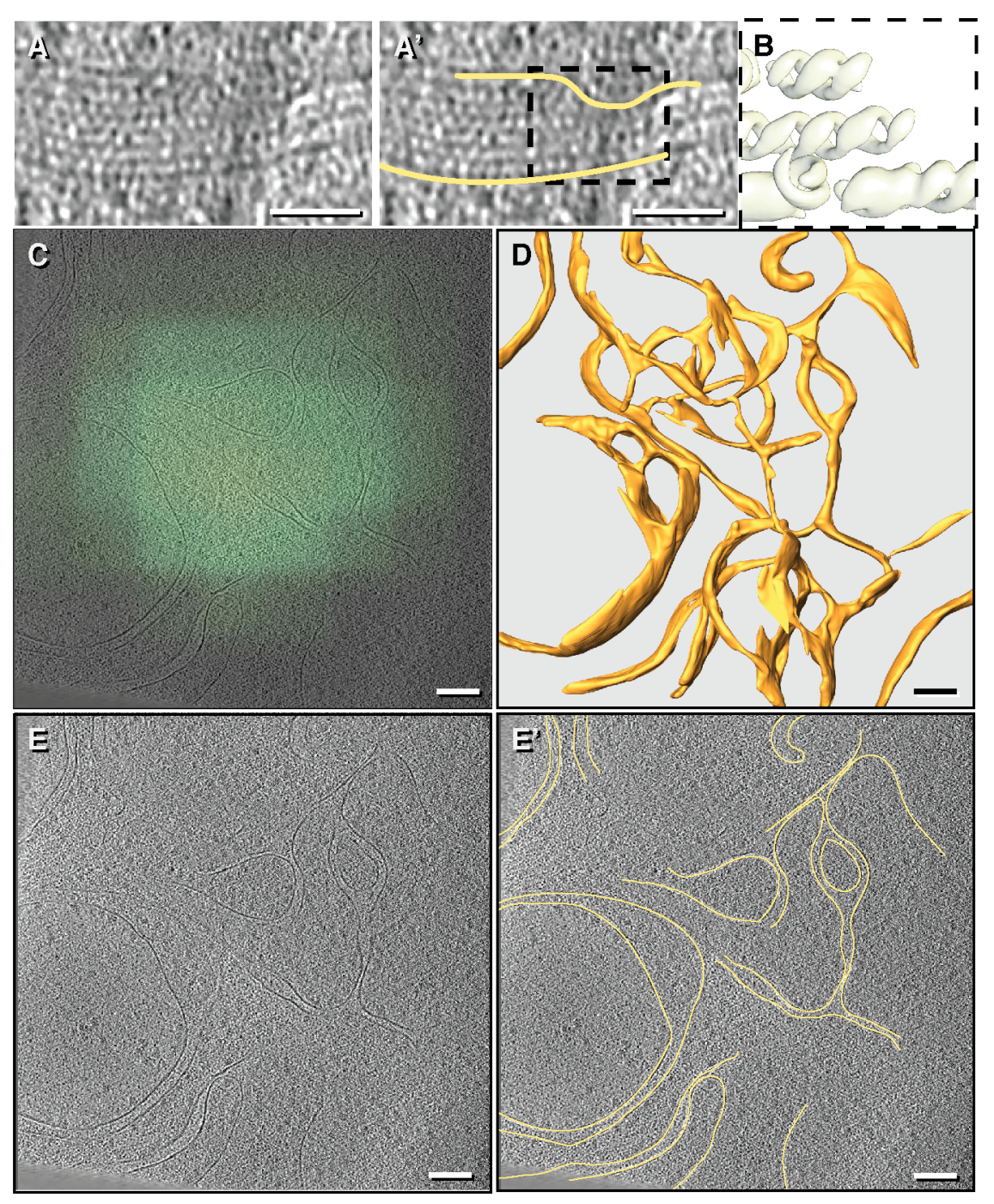

Fig. S16. Anomaly in IRE1 $\alpha$ subdomains structures.

(A, A') An example of an IRE1 $\alpha$ subdomain tube containing multiple juxtaposed IRE $\alpha$-LD filaments not separated by membrane (yellow). Scale bar $=20 \mathrm{~nm}$ (B) An isosurface of the averaged density mapped back into this region. (C) Overlay of cryo-light microscopy image and a representative slice of the reconstructed tomogram showing colocalization of IRE1 $\alpha-\mathrm{mNG}$ foci to cell sub-regions with complex membrane topology and irregular inter-membrane diameters. Scale bar $=100 \mathrm{~nm}$. (D) Segmentation of this putative precursor of IRE1 $\alpha$ subdomains with regular tube diameter, showing thin tubes connected by 3-ways junctions and enrichment of ER-fenestrations. (E, E') An example z slice of the membrane structures observed. Scale bars $=100 \mathrm{~nm}$. 


\section{Movie S1.}

IRE1 $\alpha-m$ NeonGreen foci in stressed Mouse Embryonic Fibroblasts localize to narrow IRE1 $\alpha$ subdomain tubes.

\section{Movie S2.}

IRE $1 \alpha-m$ NeonGreen foci in stressed U-2 OS cells localize to narrow IRE $1 \alpha$ subdomain tubes. ER tubules and sheet membranes are segmented in orange. IRE1 $\alpha$ subdomain tubes are shown in yellow. Other cellular membranes are shown in blue. Within the narrow IRE1 $\alpha$ subdomain tubes, lumenal protein densities with helical feature can be observed by stepping through the 3D tomogram. The lumenal double helices, resolved by subtomogram averaging, are flexible and are consistent with modeled helical arrays of human IRE1 $\alpha$ lumenal oligomers.

\section{Movie S3.}

An additional example of an IRE1 $1 \alpha-\mathrm{mNeonGreen}$ focus in U-2 OS cells. 